\title{
Vertical Structure and Size Distributions of Martian Aerosols from Solar Occultation Measurements
}

\author{
E. Chassefière and J. E. Blamont \\ Service d'Aéronomie du CNRS, BP 3,91371 Verrières-le-Buisson Cedex, France \\ V. A. Krasnopolsky and O. I. Korablev \\ Space Research Institute, Profsoyuznaya 84/32, 117810 Moscow GSP-7, USSR \\ S. K. Atreya \\ Department of Atmospheric, Oceanic, and Space Sciences. University of Michigan, Ann Arbor, Michigan 48109-2143 \\ AND \\ R. A. WEST \\ Earth and Space Science Division, Jet Propulsion Laboratory, California Institute of Technology, 4800 Oak Grove Drive, Pasadena, \\ California 91109
}

Received September 18, 1991; revised February 13, 1992

Solar occultations performed with a spectrometer on board the Soviet spacecraft Phobos 2 (Blamont et al. 1991) provided data on the vertical structure of the Martian aerosols in the equatorial region $\left(0^{\circ}-20^{\circ} \mathrm{N}\right.$ latitude) near the northern spring equinox $\left(L_{\mathrm{S}}=0^{\circ}-20^{\circ}\right)$. All measurements were made close to the evening terminator. Five clouds were detected above $45 \mathrm{~km}$ altitude and their vertical structure recorded at six wavelengths between 0.28 and $3.7 \mu \mathrm{m}$. They have a small vertical extent $(3-6 \mathrm{~km})$ and a vertical optical depth less than 0.03 . The thermal structure, as derived from saturated profiles of water vapor observed by our instrument in the infrared, does not allow the $\mathrm{CO}_{2}$ frost point to be reached at cloud altitude, strongly suggesting that cloud particles are formed of $\mathrm{H}_{2} \mathrm{O}$ ice. Under the assumption of spherical particles, a precise determination of their effective radius, which varies from cloud to cloud and with altitude, is obtained and ranges from 0.15 to $0.85 \mu \mathrm{m}$; an estimate of the effective variance of the particle size distribution is $\sim 0.2$. The number density of cloud particles at the peak extinction level is $\sim 1 \mathrm{~cm}^{-3}$. Dust was also observed and monitored at two wavelengths, 1.9 and $3.7 \mu \mathrm{m}$, on nine different occasions. The top of the dust opaque layer, defined as the level above which the atmosphere becomes nearly transparent at the wavelengths of observation, is located near $25 \mathrm{~km}$ altitude, with variations smaller than $\pm 3 \mathrm{~km}$ from place to place. The scale height of dust at this altitude is $\mathbf{3}-\mathbf{4} \mathbf{~ k m}$. The effective radius of dust particles near the top of the opaque layer is $0.95 \pm 0.25$ $\mu \mathrm{m}$ and increases below with a vertical gradient of $\sim 0.05 \mu \mathrm{m}$ $\mathrm{km}^{-1}$. Assuming that particles are levitated by eddy mixing, the eddy diffusion coefficient, $K$, is found to be $\sim 10^{6} \mathrm{~cm}^{2} \mathrm{sec}^{-1}$ at 25 $\mathrm{km}$ and $10^{5}-10^{6} \mathrm{~cm}^{2} \mathrm{sec}^{-1}$ at $50 \mathrm{~km}$ using, respectively, dust and cloud observations. An effective variance of $0.25( \pm 50 \%)$ for the dust size distribution is obtained on the basis of a simple theoretical model for the observed vertical gradient of the effective radius of dust particles. Three clouds observed by Viking at midlatitude during the northern summer are reanalyzed. The analysis gives $K \approx 10^{6} \mathrm{~cm}^{2} \mathrm{sec}^{-1}$ below $50 \mathrm{~km}$ altitude and at least $10^{7} \mathrm{~cm}^{2} \mathrm{sec}^{-1}$ above. Since the clouds seen from Phobos 2 are observed at twilight, which coincides with the diurnal maximum of the ambient temperature, they can be assumed to be in a steady state. If their thermodynamic state were to vary quickly during the day, our optical thickness at twilight would correspond to unrealistic values in earlier hours when the temperature is lower. Clouds are well fitted by theoretical profiles obtained assuming the steady state. An atmospheric temperature of $165-170 \mathrm{~K}$ at $\sim 50 \mathrm{~km}$ is inferred. The negative temperature gradient above the cloud is large $(1.5-2 \mathrm{~K}$ $\mathrm{km}^{-1}$ ). A parallel is established between these thin clouds and the polar mesospheric clouds observed on Earth. It is shown that upwelling in equatorial regions at equinox could be a significant factor in levitating cloud particles. 1992 Academic Press, Inc.

\section{INTRODUCTION}

Prior to the Phobos mission, Martian aerosols were observed by several instruments, placed either on board an orbiter (Mariner 9, Viking orbiters) or on the surface of Mars (Viking landers). A number of reflectance profiles were obtained with the imaging cameras on Mariner 9 
(Anderson and Leovy 1978), essentially in southern summer. These images were acquired under specific conditions, i.e., during the peak and decay phases of a global dust storm, with the top of the dust opaque layer near 40 $\mathrm{km}$ altitude. High opacities prevented the observation of the solid edge of the planet, resulting in uncertainties in the estimate of absolute elevations. Clouds were sometimes observed $\sim 10 \mathrm{~km}$ above the dust. High limb clouds were studied also with the help of Viking Orbiter images (Jaquin et al. 1986). The data cover a wide range of seasons and locations. In many cases, one or more haze layers below $90 \mathrm{~km}$ altitude are observed. The maximum aerosol elevation at low latitude fluctuates during the year with a minimum $(\sim 40 \mathrm{~km})$ and a maximum $(\sim 75 \mathrm{~km})$, respectively, near the northern winter and summer solstices. The Viking lander imaging cameras provided information on aerosols below $30 \mathrm{~km}$ complementary to the limb measurements (Pollack et al. 1979). The main source of the nondiurnally varying component of the opacity is the suspended micrometer-sized dust aerosol. Under normal conditions, the vertical optical thickness at the Martian surface is small, lower than 0.5 , but it may increase up to $\sim 3$ during global dust storms. The complex refractive index deduced from the angular variation of sky brightness presents a minimum near $0.8 \mu \mathrm{m}$, indicative of magnetite $\mathrm{Fe}_{3} \mathrm{O}_{4}$ with a volume mixing ratio of $\sim 1 \%$. Anatase (titanium dioxide, $\mathrm{TiO}_{2}$ ) has been suggested in order to explain the shape of the Mariner 9 absorption spectra between 200 and $350 \mathrm{~nm}$ wavelength (Pang and Ajello 1977). Anatase was later observed by the Viking lander X-ray fluorescence spectrometer (Clark et al. 1982) at a proportion of $\sim 1 \%$, together with several other metallic oxides.

Several specific questions related to the presence of aerosols in the Martian atmosphere have been recently addressed, e.g., the possible role of heterogeneous chemistry in the stability of the Martian atmosphere (Atreya and Blamont 1990). The value of the eddy diffusion coefficient may be inferred from the vertical structure of aerosols using a model in which eddy mixing of the particles is balanced by their gravitational settling. A comprehensive review of eddy diffusion coefficients derived from observations has been done by Krasnopolsky (1986). Values as large as $10^{8} \mathrm{~cm}^{2} \mathrm{sec}^{-1}$ have been found during global dust storms. Under normal conditions, a value of $10^{6} \mathrm{~cm}^{2} \mathrm{sec}^{-1}$ has been frequently suggested. A recent analysis of clouds observed from the Viking Orbiter (Kahn 1990) leads to the conclusion that the eddy diffusion coefficient $\mathrm{K}$ is small up to $60 \mathrm{~km}$ altitude, with a value of $\sim 10^{5} \mathrm{~cm}^{2} \mathrm{sec}^{-1}$. An error in the code of Kahn was detected (Kahn, personal communication) and the low values found from the first analysis were somewhat revised, with a new range of $\sim 10^{5}-10^{6} \mathrm{~cm}^{2} \mathrm{sec}^{-1}$ (Kahn 1991). From an estimate of the ice particle radii through a modeling of clouds, Kahn shows that the settling time of particles is rather short, about 1 Martian day, and that the hazes could play an important role in the Mars water cycle. Atmospheric water could be transported as ice down to the ground, with an increased adsorption efficiency in the regolith. This mechanism might play an important role in late northern summer, when a rapid decrease in atmospheric water vapor is observed by the Viking Orbiter MAWD (Mars Atmospheric Water Detector) experiment (Jakoski and Farmer 1982).

Analysis and modeling of clouds and the estimate of the relative importance of heterogeneous processes require an accurate knowledge of the particle sizes. The settling velocity of ice particles, which affects the shape and the characteristic times (formation, recycling of water and dust) of the cloud, depends on their size, which is a crucial parameter of the model. The sticking probability of a molecule in the presence of ice or dust aerosols depends on their total surface area, which cannot be estimated without simultaneous measurements of the radius and the number density of particles. Moreover, photochemical and condensation processes are expected to be extremely variable with altitude and presently there is a lack of vertical profiles of the atmospheric opacity. The purpose of the present paper is to present new observations of the Martian aerosols obtained using the solar occultation technique on the Soviet probe Phobos 2. We focus on five observations of a thin cloud layer at high altitude $(\sim 50$ $\mathrm{km})$ and nine observations of the dust at lower altitude $(\sim 10-25 \mathrm{~km})$, all made at low latitude $\left(0^{\circ}-20^{\circ} \mathrm{N}\right)$ near the northern spring equinox. The seasonal date $L_{S}$ of observations is in the range $0^{\circ}-20^{\circ}$. Clouds are observed at several wavelengths between 0.28 and $3.7 \mu \mathrm{m}$. The dust is observed only at two wavelengths with the infrared channels of the instrument $(1.9$ and $3.7 \mu \mathrm{m}$ ) because regions below $\sim 30 \mathrm{~km}$ altitude are not sounded by the UV-NIR line of sight. This lack of data results from an error in the software of the pointing device which led to an erroneous preliminary interpretation of the data (Blamont et al. 1989). The behavior of the instrument is now understood and the defects are taken into account properly, as discussed in the second paper by Blamont et al. (1991), referred to as PSP in the following. Nevertheless, the inclusion of infrared spectra of clouds allows us now to substantially improve our estimates of particle radii and number densities.

\section{THE DATA}

\section{Description of the Instrument}

The instrument has been designed for solar occultation measurements of the Martian atmosphere. It is composed of three different dispersive systems: a spectrograph using a holographic grating providing a spectrum of 512 elements in the spectral range $215-328 \mathrm{~nm}$ with a mean reso- 
lution of $1.8 \mathrm{~nm}$; a Fabry-Perot interferometer placed behind a predispersing grating providing, with a resolution of 150,000 , two spectra of 80 elements each with spectral ranges of 0.2 and $0.3 \mathrm{~nm}$ centered, respectively, at 760 and $936 \mathrm{~nm}$; a grating spectrograph providing two spectra of 24 elements each, in the ranges $5290-5370 \mathrm{~cm}^{-1}$ and $2707-2740 \mathrm{~cm}^{-1}$ with a resolution of $10^{3}$. A detailed description of the instrument may be found in previous papers [Blamont et al. (1989), PSP for the UV spectrometer and the NIR interferometer; Krasnopolsky et al. (1989) for the IR spectrometer]. Its principle is to measure from the Phobos orbit the spectrum of the Sun, modified by atmospheric extinction, during sunset and sunrise. Because of the large apparent diameter of the Sun projected on the occultation plane $(\sim 60 \mathrm{~km}$ in circular orbit), it is necessary to select a small part of the Sun. The UV-NIR spot has an angular diameter of $1 \mathrm{arcmin}$, or $\sim 3 \mathrm{~km}$ vertical resolution, and is located near the center of the solar disk. The IR field is about twice as large and its line of sight is shifted by 8.5 arcmin, or $\sim 20 \mathrm{~km}$, relative to the previous one. It is therefore located near the edge of the solar disk. The detectors of all spectrographs are linear arrays of photodiodes, which detect all the spectral elements simultaneously. For measurements considered in the present paper, sampling times are generally $0.5,1$, and $2 \mathrm{sec}$ for, respectively, IR, UV, and NIR channels, corresponding to vertical excursions of the line of sight of, respectively, 1,2 , and $4 \mathrm{~km}$ under nominal conditions. In some cases there is an increase of the integration time during occultation following a preselected sequence and the last spectra before sunset are obtained with integration times of 2, 3, or $4 \mathrm{sec}$. Due to the pointing error, an oscillation of the line of sight is observed in two cases (see PSP); the vertical excursion during the sampling time may vary by more than a factor of 2 .

The instrument operated from February 8 to March 26 (the Martian equinox occurred on February 17). Thirtyeight solar occultations were observed during ingress and one was observed during egress. Results reported here were made when the spacecraft was in a circular orbit (planetocentric radius, $9800 \mathrm{~km}$; period, $8.1 \mathrm{~h}$ ). The latitude of the intersection of the Sun-spacecraft axis with the surface of Mars varied from $-11^{\circ}$ on February 8 to + $20^{\circ}$ on March 26, and the distance between the spacecraft and the limb was $10^{4} \mathrm{~km}$. The data set examined in this paper consists of four type 1 and nine type 2 occultations obtained during ingress at sunset between February 20 and March 26. Type 1 and type 2 refer to two different modes of pointing, as explained in PSP. Each session is characterized by seven numbers indicating the time period from the launch (day, hour, minutes). For simplicity, occultations will be identified in the text by the three first numbers (day of occultation: the first session on February 20 is $\mathrm{S} 221$ ). The minimum altitude reached by the
UV-NIR line of sight is $\sim 40 \mathrm{~km}$ for type 1 and $\sim 30 \mathrm{~km}$ for type 2. Due to the geometry of the optics, the IR line of sight is $\sim 20 \mathrm{~km}$ below the UV-NIR line of sight in the Martian atmosphere. In nine cases (type 2) a minimum altitude of $\sim 10 \mathrm{~km}$ is reached and the extinction of the sunlight by the atmospheric dust at this level is almost total. Thus, nine complete occultations were obtained in the two IR channels.

\section{Treatment of Data}

The treatment of UV and NIR signals has been discussed elsewhere (PSP). Because of instrumental defects, measured UV spectra consist of a superimposition of several components. Due to this complication and because of the rapid degradation of the optics at wavelengths shorter than $0.25 \mu \mathrm{m}$, we used only solar line depths for signal (Blamont et al. 1989). The signal is corrected for centerto-limb darkening when the loss of pointing occurs and split into its two main components (UV and stray-light) at four wavelengths $(0.270,0.277,0.283$, and $0.320 \mu \mathrm{m})$. The wavelength range where the data are pure stray light can be used as a signal at $0.40-0.50 \mu \mathrm{m}(\sim 0.43 \mu \mathrm{m})$. In the present study, the value at $0.32 \mu \mathrm{m}$ was disregarded because of the weakness of the corresponding solar line. In certain cases, there is a lack of information for some of the three other lines due to telemetry errors or saturation of the detector. It was decided to do the average between the available lines in order to reduce the noise and obtain one single reliable point at $\sim 0.28 \mu \mathrm{m}$. The stray light provides another point in the NUV $(\sim 0.43 \mu \mathrm{m})$. Two additional points are obtained in the NIR by integrating the signal in the two channels of the interferometer $(0.76$ and $0.94 \mu \mathrm{m}$ ).

The behavior of the signal at 1.9 and $3.7 \mu \mathrm{m}$ is strongly affected by pointing problems. As previously mentioned, the IR line of sight is located under nominal conditions near the solar edge, at 8.5 arcmin from the UV-NIR line of sight and on the bisecting line of the two pointing axes (see Fig. 2 in PSP). In the geometrical configuration prevailing at the time of the Phobos observations, the IR line of sight was located below the UV-NIR line of sight during the occultation phase, shifted by $\sim 20 \mathrm{~km}$ in the occultation plane toward the surface of Mars. Fortuitously, it is at about the same altitude as the lowest pointing eye denoted $\mathrm{L}$ (see Fig. 2 in PSP). For reasons that were explained in PSP, any atmospheric darkening in the $\mathrm{L}$ pointing eye produced a decrease of the descent velocity of the line of sight. Because the same atmospheric region is seen at the same time in the $L$ eye and the IR field of view, any decrease of the IR signal is systematically accompanied by a perturbation of the pointing, which makes difficult the restitution of the altitude. In the presence of a cloud, an oscillation of the line of sight is ex- 
pected (see Fig. 3 in PSP), occuring simultaneously with the detection of the cloud by the IR spectrometer. The surprising evolution of the IR signal in some cases, especially during one of the type 1 occultations (S230), analyzed in appendix B, may be clearly interpreted as the signature of this effect, although a detailed analysis indicates unexplained features, probably due to some instrumental defects in the IR subsystem. Adding to the two IR signals, integrated over their whole spectral ranges, we consider the $\mathrm{CO}_{2}$ transmission in the band at $5313 \mathrm{~cm}^{-1}$ which allows us to retrieve altitudes.

Because of the geometry, the type 2 configuration is the most favorable. As shown in PSP, the deepest level reached by the UV-NIR line of sight is about $10 \mathrm{~km}$ lower for type $2(\sim 30 \mathrm{~km})$ than for type $1(\sim 40 \mathrm{~km})$. For type 2 , there is a time delay of some seconds, corresponding to a vertical excursion of $\sim 10 \mathrm{~km}$, during which extinction is observed in IR channels and the pointing system still works in the nominal way. Thanks to this short delay, the dust layer was observed nine times down to an altitude of $10-15 \mathrm{~km}$, depending on the geometry. From an analysis of the pointing fluxes, it may be concluded that the three first type 2 occultations are made without any substantial loss of pointing. Due to the evolution of the geometry from February to the end of March 1989, the situation is less good for later type 2 occultations. Nevertheless, there are no major pointing problems for dust observations in the IR. On the other hand, four among the five clouds detected are of type 1 and it is necessary to take into account the loss of pointing when analyzing the IR data. The treatment of the IR data, including the restitution of altitudes from $\mathrm{CO}_{2}$ absorption, is discussed in Appendix A for type 2 and Appendix B for type 1.

\section{RADIUS AND NUMBER DENSITY OF CLOUD PARTICLES}

\section{Preparation of Data}

In order to treat simultaneously the data obtained by the three spectrometers of the experiment and to compare extinction coefficients at the different selected wavelengths $(0.28 \mu \mathrm{m}, 0.43 \mu \mathrm{m}-\mathrm{UV} ; 0.76 \mu \mathrm{m}, \quad 0.94$ $\mu \mathrm{m}-\mathrm{NIR} ; 1.9 \mu \mathrm{m}, 3.7 \mu \mathrm{m}-\mathrm{IR})$, it is necessary to make integration times and spatial fields of view as similar as possible. One may easily bring together UV and NIR measurements which are obtained along the same line of sight. Because NIR data are acquired with a sampling time $(2 \mathrm{sec})$ larger than UV data $(1 \mathrm{sec})$, the temporal resolution in the UV has been degraded, grouping measurements two by two. In some short periods, e.g., at the end of the very long S236 observation, there is an increase of the integration time in the UV-NIR following a preprogrammed sequence with a loss of vertical resolution. During a time of $2 \mathrm{sec}$, the line of sight covers $\sim 4 \mathrm{~km}$ in nominal pointing conditions. The angular field of view is

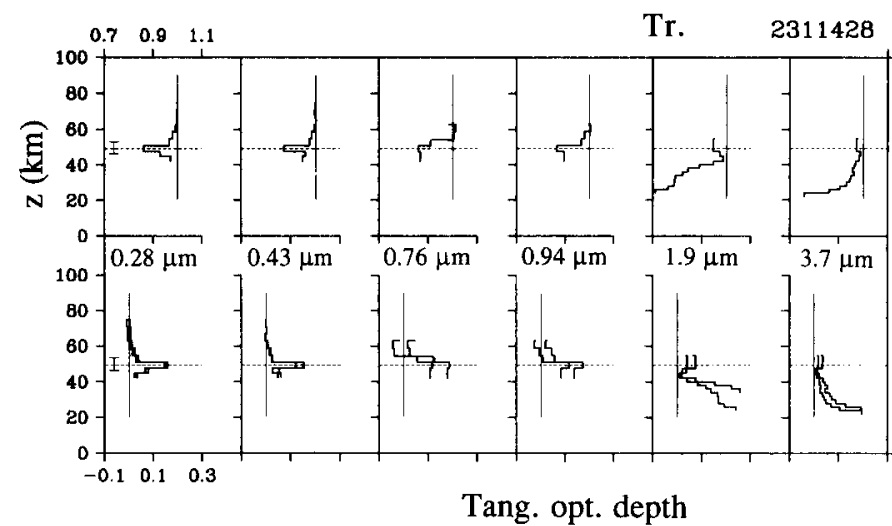

FIG. 1. Vertical profiles of transmission (upper part) and tangential optical thickness (lower part) at the six wavelengths of the experiment for $\mathrm{S} 231$. Units for the transmission are given at the top of the left upper figure $(0.7$ to 1.1$)$, those for the optical thickness at the bottom of the left lower figure $(-0.1$ to 0.3$)$. The wavelength increases from the left to the right. It is indicated in micrometers. The error bar on optical depths is represented by two extreme profiles. An horizontal dashed line is placed at the center of the peak extinction layer and the error bar on the altitude of the cloud is indicated on the left.

1 arcmin, i.e., $\sim 3 \mathrm{~km}$ in the Martian atmosphere. The resulting vertical resolution is therefore $\sim 5 \mathrm{~km}$, which is also the diameter of the IR projected field of view. The same region is not seen at the same time in the UV-NIR and in the IR because of the vertical shift of $\sim 20 \mathrm{~km}$ between the two lines of sight in the occultation plane. The value of the shift deduced from experimental specifications may be checked using the peak extinction levels of the two thickest clouds. A residual error of $1.5 \mathrm{~km}$ is found. This error is linked to the uncertainty in the relative positions of the two lines of sight. IR profiles have been shifted by $1.5 \mathrm{~km}$ upward. IR transmissions, obtained each $0.5 \mathrm{sec}$, are averaged over time intervals corresponding to one UV-NIR measurement.

Clouds are also identified by the three numbers of the day, e.g., C231 is the cloud observed during session S231. Figures 1 to 3 show the vertical profiles of the transmission as seen by the instrument and the deduced profiles of the tangential optical thickness at the six wavelengths of interest for three of the five observed clouds. C236 (Fig. 2) and C251 (Fig. 3) are the two biggest clouds observed by our instrument. C231 (Fig. 1) and C245 are more diffuse. For the very diffuse C237, the spectrum in the NIR has been lost because of telemetry errors and no reliable estimate of the particle radius may be done. As quoted in Appendix B, there is probably a sixth cloud (C230, see Fig. B1). Clouds are therefore observed 6 times out of a total of 38 , i.e. in $15 \%$ of cases. The error bar on the tangential optical thickness is estimated taking into account the different sources of uncertainty: (a) $0.28 \mu \mathrm{m}$, random noise, reference level (zero optical depth); (b) 


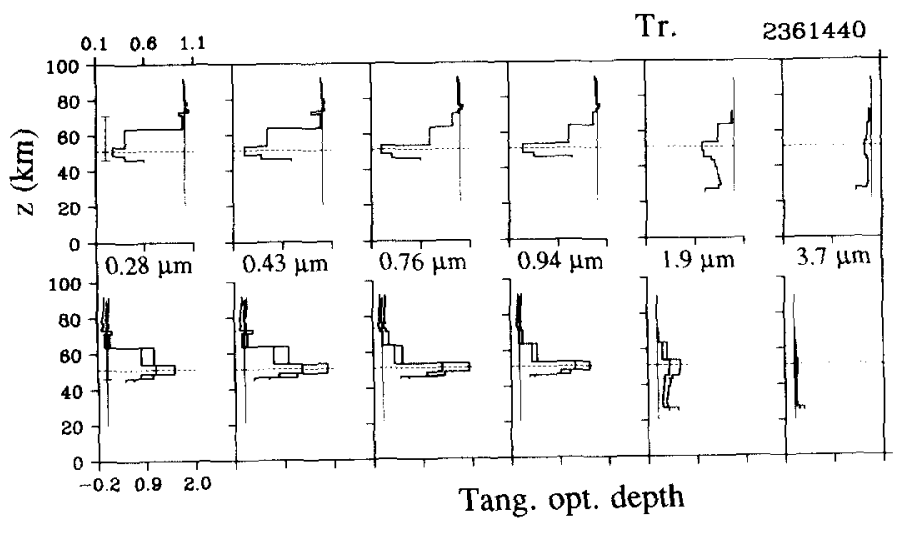

FIG. 2. Same as Fig. 1 for S236. The great width of some layers is due to the large oscillation of the line of sight.

$0.43 \mu \mathrm{m}$, random noise, reference level, superimposition on the main UV beam of a secondary beam (see Fig. 3 in PSP) (the relative weight of the secondary image of the Sun, shifted by $1-2 \mathrm{~km}$ with respect to the primary one, is assumed to lie between 25 and $75 \%$ and the profile is deconvoluted under these two extreme assumptions); (c) $0.76 \mu \mathrm{m}, 0.94 \mu \mathrm{m}$, random noise, reference level; (d) 1.9 $\mu \mathrm{m}, 3.7 \mu \mathrm{m}$, random noise, reference level, uncertainty due to the use of corrective functions estimated to be $\pm 30 \%$. The two corresponding extreme profiles of the tangential optical thickness are shown in Figs. 1 to 3. Although it is difficult to treat errors in statistical terms due to the instrumental bias, these extreme profiles may be thought of as statistical error bars at the $2 \sigma$ level. The strong disturbance of the vertical sampling for $\mathrm{C} 236$ is due to the large oscillation of the line of sight when the cloud goes through pointing eyes (see Figs. 3 and 4 in PSP). All clouds are nearly transparent at $3.7 \mu \mathrm{m}$ but two of them (C236, C251) are definitely observed at $1.9 \mu \mathrm{m}$. C245, the only cloud obtained in type 2 , is never seen in the IR

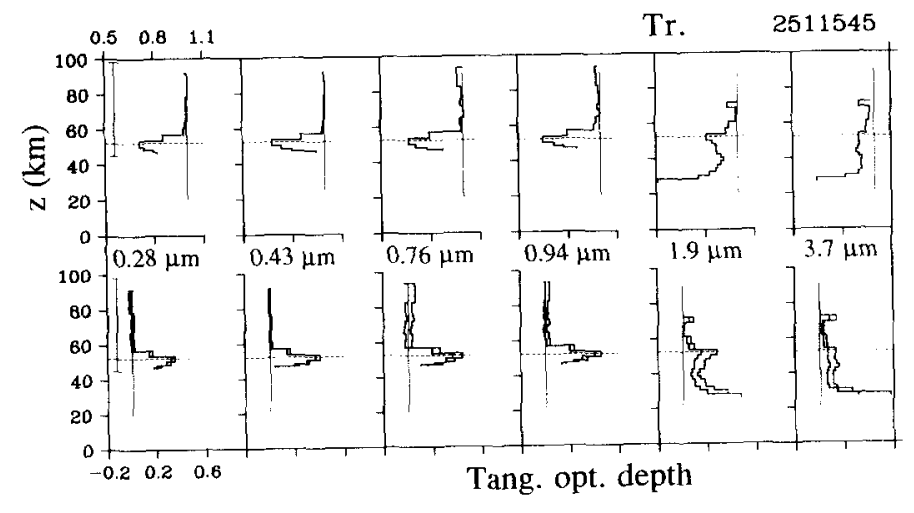

FIG. 3. Same as Fig. 1 for S251. because at the time of the first measurement, the IR line of sight was already below the cloud level. There is a lack of some data in NIR because of telemetry. We will analyze the spectrum of one layer (peak extinction layer, denoted by PL hereafter) for C231, three layers (PL, the top layer, TL, above PL and the bottom layer, BL, below PL) for $\mathrm{C} 236$, one layer for C245 (PL), and four layers for C251 (PL, TL, BL, and the layer BBL below BL).

\section{Estimate of the Particle Radius in Clouds}

In a first approximation, the size distribution of cloud particles may be represented by a simplified $\gamma$ law of the form

$$
\frac{d n}{d r}=k r \frac{1-3 b}{b} e^{-\frac{r}{a b}}
$$

where $a$ is the effective radius (area weighted radius) and $b(<0.5)$ the effective variance of the distribution (Hansen and Travis 1974). The effective variance may be defined as the standard deviation of the distribution normalized by the effective radius. A typical value of $b$ for terrestrial nonconvective clouds is $0.1-0.2$ (e.g., Mason 1971). Mie theory was used to derive the particle radius from the wavelength dependence of the particle optical thickness. Mie theory is strictly valid only for spherical particles, while Martian aerosols (ice or dust particles) are probably multifaceted crystals. West (1992) investigated the effects of nonsphericity on particle sizing for aggregate particles, although in a size regime smaller than that found in this work. It is not clear that calculations for aggregate particles are relevant to Mars, although West's calculations can be used to estimate errors made by the assumption of spheres. For this purpose, extinction cross-sections for an aggregate particle have been calculated at the wavelengths of the observations. They have been treated as if they were real data using the retrieval algorithm described later, which is based on tables for spheres. The best fit is obtained for an effective radius of $0.15 \mu \mathrm{m}$, only slightly larger than the true equivalent area radius of the aggregate particle, $-0.14 \mu \mathrm{m}$. Although the absolute extinction cross-sections of spheres and aggregate particles may differ by a factor of $\sim 2$ (see Fig. 3 of West's paper), the retrieval of the effective radius, which is based on the shape of the particle extinction spectrum, is only slightly biased in the presence of small irregular particles.

We recognize the desire to perform the particle size retrievals using calculations for nonspheres, but practical limitations prevented us from doing so. The derived wavelength dependence of the optical thickness reaches a maximum at visible wavelengths for many of our data samples, indicating particle radius comparable to or larger than the shortest wavelengths. Optical properties of these large 
particles could not be computed accurately with methods such as the discrete dipole approximation (West 1992) available to us during this work. In addition, the problem of deciding which nonspherical shapes to use in the calculations must be resolved, with no data available on the shapes of particles in the Martian atmosphere. While further progress in this area is envisioned with more powerful algorithms now available, the task is a formidable one and not really necessary for this work. Future investigators wishing to reanalyze our results for nonspherical particles can do so by finding solutions which have the same wavelength dependence as the spherical distributions derived here.

Calculations of the extinction cross-section of water ice particles supposed spherical have been made for a monodisperse distribution (one single size) with a particle radius in the range from 0.1 to $4 \mu \mathrm{m}$. The step is $0.1 \mu \mathrm{m}$ from 0.1 to $1 \mu \mathrm{m}, 0.2 \mu \mathrm{m}$ from 1 to $2 \mu \mathrm{m}$ and $0.4 \mu \mathrm{m}$ from 2 to $4 \mu \mathrm{m}$. The shape of the opaque layer, with a pronounced peak and a sharp decrease of the extinction coefficient above, cannot be produced by dust, which is observed $\sim 25 \mathrm{~km}$ lower in IR channels. On the other hand, as shown in the modeling section, this type of shape is perfectly consistent with the presence of a condensation layer. The vertical profile of the temperature has been deduced from $\mathrm{H}_{2} \mathrm{O}$ measurements in the IR (Krasnopolsky et al. 1991). The temperature decreases from $\sim 180 \mathrm{~K}$ at $15 \mathrm{~km}$ altitude to $\sim 150 \mathrm{~K}$ at $40 \mathrm{~km}$ altitude. The $\mathrm{CO}_{2}$ frost point temperature is $148 \mathrm{~K}$ at the ground level and decreases down to $\sim 125 \mathrm{~K}$ at $40 \mathrm{~km}$ altitude. The atmospheric temperature at the cloud altitude, as derived from IR measurements, is therefore $\sim 25 \mathrm{~K}$ above the $\mathrm{CO}_{2}$ condensation temperature. This disagreement could be understood if one assumes that the altitude of the cloud layer is underestimated by $\sim 30 \mathrm{~km}$. Indeed, the $\mathrm{CO}_{2}$ frost point temperature vertical profile and the thermal profile obtained by extrapolating the observed profile above $40 \mathrm{~km}$ meet at $\sim 80 \mathrm{~km}$ altitude. Such a possibility may be firmly rejected since, at least for C231 (Fig. 1), C237, and C245, the altitude of the cloud is lower than $\sim 55 \mathrm{~km}$. This result strongly suggests that the clouds observed at high altitude are composed of water ice. The convolution of crosssections by the radius distribution function [Eq. (1)] was made for $b$ in the range $0.05-0.45$ (step of 0.05 ). The theoretical spectrum of water ice at the six wavelengths of interest has been obtained in this matter for all combinations $(a, b)$ with $0.1 \mu \mathrm{m}<a<4 \mu \mathrm{m}$ and $0.05<b<0.45$. Theoretical and observed spectra are normalized at 0.28 $\mu \mathrm{m}$ by dividing the values of the cross-section or the optical thickness at the different wavelengths by the value at $0.28 \mu \mathrm{m}$. The particle number density will be estimated next with a better vertical resolution by dividing the extinction coefficient at $0.28 \mu \mathrm{m}$ by the extinction cross section derived from the estimate of the particle radius.
Each observed spectrum is compared to each one of the theoretical spectra of the complete series, and the square deviations weighted by error bars summed over the five free wavelengths (excluding $0.28 \mu \mathrm{m}$ because of the normalization) for each value of $(a, b)$. In this way, the mean standard deviation in units of one error bar may be derived as a function of $a$ and $b$.

The vertical profiles of the cloud tangential optical thickness may be inverted at each wavelength to retrieve the corresponding local extinction coefficient. A simple "onion peeling" technique is used in the present work (see Appendix C). In this way, a spectrum of the extinction coefficient, called for simplicity inverted spectrum, may be associated with any original spectrum of the tangential optical thickness. On a trial basis, both initial and inverted spectra were fitted by theoretical extinction spectra of ice particles in order to characterize the size distribution of cloud particles. The fits are certainly not as good when using inverted spectra. Two reasons for this may be identified: (1) There are pointing problems which require a complex treatment of all data in order to eliminate instrumental effects and some small systematic errors are amplified through inversion. (2) The observed clouds are very thin and therefore they may present some horizontal variations of altitude or opacity which invalidate the classical onedimensional inversion techniques. No rigorous corrections can be applied in this case. Although the general vertical profile of the extinction coefficient in the cloud is rather well estimated from a simple one-dimensional inversion, the spectral shape is not well retrieved because it is very sensitive to the radius of particles. Because of the above-mentioned difficulties it was decided to work on the horizontal optical thickness rather than the local opacity to determine the particle radius. As is obvious, this results in no bias on the radius in the top layer TL. On the other hand, there is a slight bias for the peak layer PL since, when sounding it, the line of sight intersects TL. Nevertheless, due to both the geometry and the rapid decrease of the particle number density from PL to TL, the contribution of TL to the extinction is weak. The estimated radius for PL is shifted toward the radius of particles in TL by no more than $\sim 10 \%$ of the difference between them. As seen hereafter, layers below PL disappear when inverting spectra, showing that the only contribution to the measured opacity when the tangential point is below the cloud is extinction by PL, at a large distance from the tangential point. Therefore any vertical sounding of the cloud below the peak in occultation conditions corresponds in fact to a horizontal excursion of the peak region at large distance from the tangential point. The radius and the variance derived from observations for layers BL, BBL give information on the horizontal variability of the particle size distribution. This information would be lost using inverted spectra. 

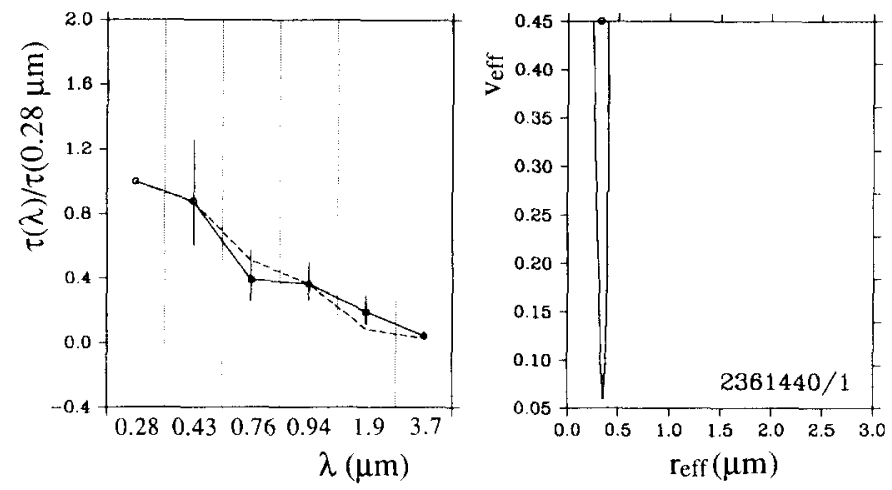

FIG. 4. On the left is shown the best fit between the predicted and the observed spectra (indicated resp. by the dashed line and the solid line with error bars) normalized at $0.28 \mu \mathrm{m}$ for the top layer TL of cloud C236. On the right is plotted in the plane $a$ (effective radius) $-b$ (effective variance) the field where the agreement is better than $2 \sigma$. The circle corresponds to the best fit.

In Figs. 4 and 5 are shown the best fits obtained for the most opaque cloud C236 (resp., TL and PL). Error bars on the observed tangential optical thickness are directly deduced from extreme profiles of Fig. 2. On the right side the point of the plane $(a, b)$ where the best fit is obtained is indicated by a small circle. The field delineated by the thick solid line is the region of the plane where the standard deviation is lower than $2 \sigma$. The thin contour, when it exists, delineates the field where agreement is realized with an accuracy better than $1 \sigma$. The estimate of the particle radius does not depend too much on the assumed value of the variance of the distribution, which is poorly constrained. The shape of the spectrum changes when moving down to the peak, from a regular decrease with wavelength characteristic of a small radius $\sim 0.3 \mu \mathrm{m}$ (Fig. 4) in TL to a flatter profile in the UV-NIR, implying a radius of $\sim 0.5 \mu \mathrm{m}$ (Fig. 5) in PL. Although C245 was not
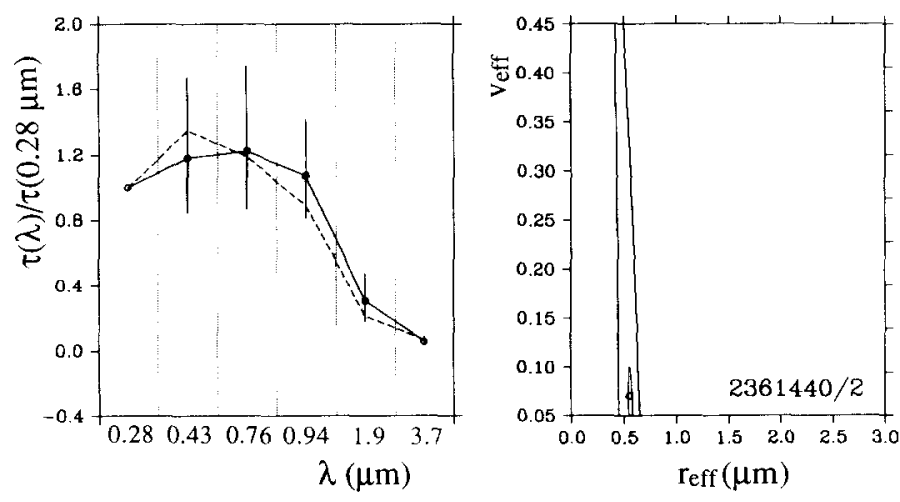

FIG. 5. Same as Fig. 4 for $\mathrm{C} 236$ (PL). On the right are plotted in the plane $a-b$ the fields where the agreement is better than $I \sigma$ (thin line) and $2 \sigma$ (thick line).
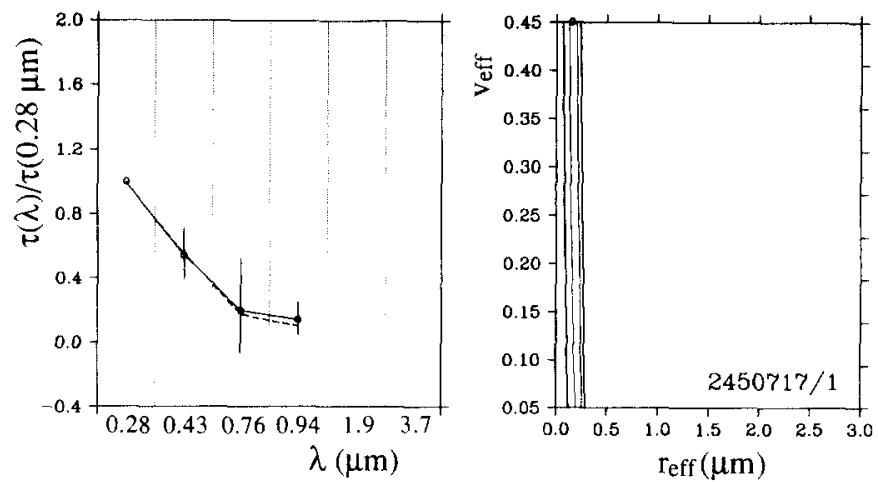

FIG. 6. Same as Fig. 5 for C245 (PL).

observed in the IR, the radius of particles in PL may be estimated rather accurately from only UV-NIR wavelengths (Fig. 6). The rapid decrease of the optical thickness with increasing wavelength is characteristic of very small particles $(\sim 0.2 \mu \mathrm{m})$. Larger particles $(0.8-1 \mu \mathrm{m})$ are observed in C25I (PL), as shown by Fig. 7. The fit is good and the effective variance well estimated $(\sim 0.2)$.

Figures 8 to 11 summarize the results found from the previous analysis. Vertical profiles of the tangential optical thickness, which is the measured quantity, and those derived by inversion (i.e., the vertical optical thickness of particles per unit atmospheric length, e.g., $1 \mathrm{~km}$ ), are plotted together. Optical profiles are given at $0.28 \mu \mathrm{m}$, where the vertical resolution is best. As previously explained, layers below the peak disappear through inversion. This effect is well marked for C231 (Fig. 8) and C251 (Fig. 11). The vertical profiles of the effective radii and effective variances are also plotted. The most opaque cloud (C236, Fig. 9) has an extinction coefficient at the peak of $\sim 4 \times 10^{-3} \mathrm{~km}^{-1}$. The optical depths of the clouds, i.e., the integral of the extinction coefficient over altitude from the top of the atmosphere, are given in Table I together with other characteristics of the clouds. These
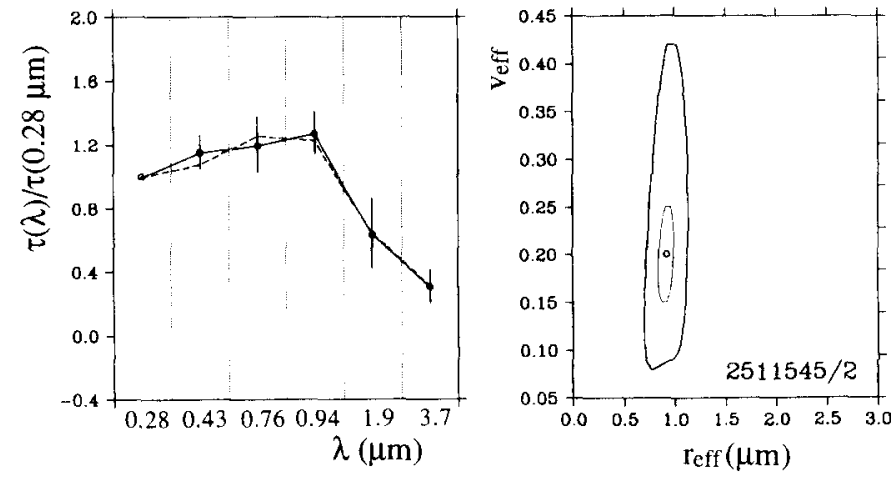

FIG. 7. Same as Fig. 5 for C251 (PL). 

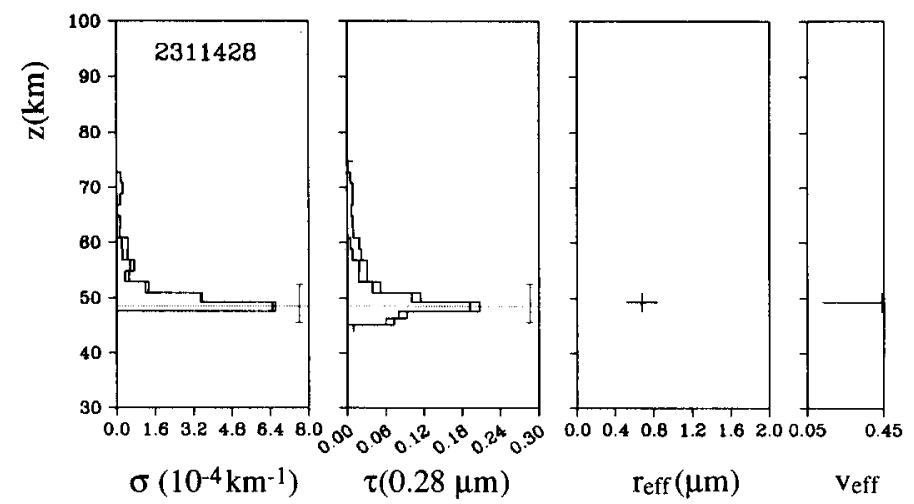

FIG. 8. From the left to the right for C231: the vertical profile of the extinction coefficient at $0.28 \mu \mathrm{m}$ in units of $\mathrm{km}^{-1}$, the vertical profile of the tangential optical thickness at $0.28 \mu \mathrm{m}$ together with the uncertainty on the altitude, the average effective radius in the altitude range indicated by the vertical error bar, and the corresponding effective variance. The vertical resolution is worse for the radius and the variance than for the extinction coefficient because the resolution was degraded in the UV in order to make the comparison with other wavelengths possible.

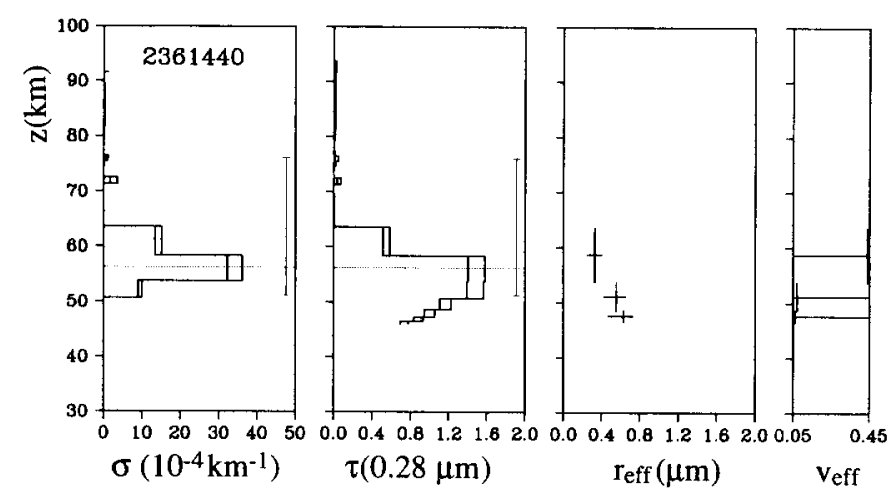

FIG. 9. Same as Fig. 8 for C236

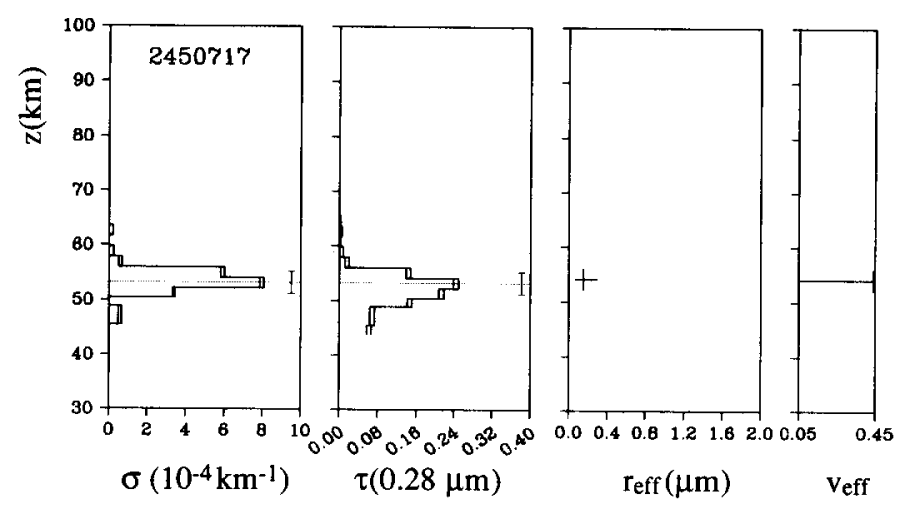

FIG. 10. Same as Fig. 8 for C245.

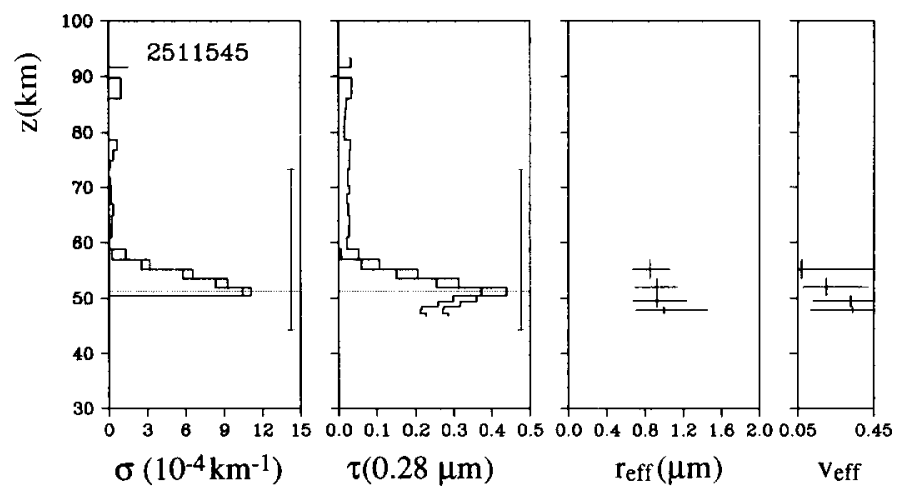

FIG. 11. Same as Fig. 8 for C251.

are weak, smaller than 0.03 . These high clouds are therefore almost completely transparent. Diverse results are obtained on the size of the particles with a dispersion of one order of magnitude on the mean effective radius $(\sim 0.1-1 \mu \mathrm{m})$ and a slight increase of the radius from the top of the cloud down to the peak. Below the peak, there is no significant increase of the radius, suggesting that the particle radius is nearly constant in the horizontal

TABLE I

Characteristics of the Five Clouds Observed by Solar Occultation from Phobos

\begin{tabular}{|c|c|c|c|c|c|c|c|c|c|c|}
\hline & $\begin{array}{c}\text { Peak } \\
\text { alditude } \\
\text { (km) }\end{array}$ & $\begin{array}{c}W \\
(\mathrm{~km})\end{array}$ & $\begin{array}{l}\int \tau_{\text {ext }} \\
\left(10^{-2}\right)\end{array}$ & $\begin{array}{c}\tau_{\text {ext }} \text { at } \\
\text { peak } \\
\left(10^{-3}\right. \\
\left.\mathbf{k m}^{-1}\right) \\
\end{array}$ & layer & $\begin{array}{c}\text { eff } \\
\text { radius } \\
(\mu \mathrm{m})\end{array}$ & $\begin{array}{l}\text { eff. } \\
\text { var. }\end{array}$ & $\begin{array}{c}\mathrm{a}_{\text {part at }} \\
\text { peak } \\
\left(\mathrm{cm}^{-3}\right)\end{array}$ & $\begin{array}{c}\int \mathrm{S} \\
\left(\mathrm{mm}^{2}\right. \\
\left.\mathrm{cm}^{-2}\right)\end{array}$ & $\begin{array}{c}N\left(10^{-4}\right. \\
\mathrm{mm}^{3} \\
\left.\mathrm{~cm}^{-2}\right)\end{array}$ \\
\hline C231 & $\begin{array}{c}48 \\
(+4,-3)\end{array}$ & 3 & $\begin{array}{r}0.23 \\
\pm 0.02 \\
\end{array}$ & $\begin{array}{r}0.65 \\
\pm 0.01 \\
\end{array}$ & peak & $\begin{array}{r}0.65 \\
\pm 0.15 \\
\end{array}$ & $>0.15$ & $\begin{array}{c}0.7 \\
\pm 0.5 \\
\end{array}$ & $\begin{array}{c}0.4 \\
\pm 0.04 \\
\end{array}$ & $\begin{array}{c}0.8 \\
\pm 0.25 \\
\end{array}$ \\
\hline \multirow[t]{2}{*}{ C236 } & \multirow[t]{2}{*}{$\begin{array}{c}56 \\
(+?-5)\end{array}$} & \multirow[t]{2}{*}{6} & \multirow[t]{2}{*}{$\begin{array}{r}2.7 \\
+0.2\end{array}$} & \multirow[t]{2}{*}{$\begin{array}{c}3.4 \\
\pm 0.2\end{array}$} & top & $\begin{array}{c}0.3 \\
\pm 0.1\end{array}$ & - & \multirow[t]{2}{*}{$\begin{array}{c}4.7 \\
\pm 3.3\end{array}$} & \multirow[t]{2}{*}{$\begin{array}{c}4.7 \\
\pm 0.2\end{array}$} & \multirow[t]{2}{*}{$\begin{array}{c}7.8 \\
\pm 2.1\end{array}$} \\
\hline & & & & & peak & $\begin{array}{r}0.55 \\
\pm 0.1 \\
\end{array}$ & - & & & \\
\hline $\mathrm{C} 237$ & $\begin{array}{c}50 \\
(+4,-2) \\
\end{array}$ & $\approx 3$ & & & & - & - & & & \\
\hline $\mathrm{C} 245$ & $\begin{array}{c}53 \\
(+2,-2) \\
\end{array}$ & 4 & $\begin{array}{c}0.36 \\
\pm 0.02 \\
\end{array}$ & $\begin{array}{r}0.79 \\
\pm 0.01 \\
\end{array}$ & peak & $\begin{array}{r}0.15 \\
\pm 0.1 \\
\end{array}$ & & $\begin{array}{r}0.5 \\
\pm 0.3 \\
\end{array}$ & $\begin{array}{r}5.3 \\
\pm 0.4 \\
\end{array}$ & $\begin{array}{r}9.7 \\
\pm 2.5 \\
\end{array}$ \\
\hline \multirow[t]{2}{*}{ C251 } & \multirow[t]{2}{*}{$\begin{array}{c}51 \\
(+?,-7)\end{array}$} & \multirow[t]{2}{*}{4.5} & \multirow[t]{2}{*}{$\begin{array}{c}0.51 \\
\pm 0.08\end{array}$} & \multirow[t]{2}{*}{$\begin{array}{c}1.1 \\
\pm 0.1\end{array}$} & top & $\begin{array}{r}0.85 \\
\pm 0.2 \\
\end{array}$ & - & \multirow[t]{2}{*}{$\begin{array}{c}0.8 \\
\pm 0.5\end{array}$} & \multirow[t]{2}{*}{$\begin{array}{c}6.1 \\
\pm 0.5\end{array}$} & \multirow[t]{2}{*}{$\begin{array}{r}12.4 \\
\pm 3.2\end{array}$} \\
\hline & & & & & peak & $\begin{array}{c}0.9 \\
\pm \mathbf{0 . 2} \\
\end{array}$ & $\begin{array}{c}0.25 \\
\pm 0.15 \\
\end{array}$ & & & \\
\hline
\end{tabular}

Note. The altitude and the width at half maximum of the cloud are reported in the first two columns. In the third and fourth columns are indicated respectively the vertical optical depth of the cloud and the maximum of the extinction coefficient at $0.28 \mu \mathrm{m}$ wavelength. Depending on the cloud, data provide information on the particle size at the top and at the peak (C236 and C251), only at the peak (C231 and $\mathrm{C} 245$ ), or no information at all (C237). The effective radius is given in the sixth column. When available, the allowed range of the effective variance is reported in the seventh column. In the three last columns are indicated respectively the number density of ice particles at peak extinction and their vertically integrated surface and volume. Particles are assumed to be spherical. 


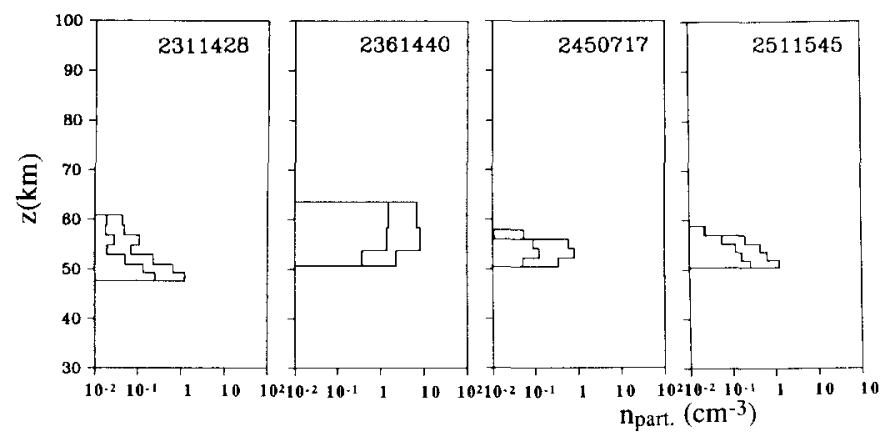

FIG. 12. Vertical extreme profiles (lower and upper limits) of the number density of ice particles in clouds.

direction. There is a definite increase of the variance with decreasing altitude for $\mathrm{C} 251$, at least at the $1 \sigma$ level. This fact could represent the signature of a horizontal variability in the effective variance of the size distribution, with a slight increase in the dispersion when moving away from the tangential point around the peak extinction altitude. In Fig. 12 are plotted on a logarithmic scale the vertical profiles of the particle number density for the four clouds previously analyzed. The number density of the ice particles is in the range of a few tenths to one per cubic centimeter (see Table I).

\section{PARTICLE SIZE AND NUMBER DENSITY NEAR THE TOP OF THE DUST OPAQUE LAYER}

In nine cases, the 10 upper kilometers of the dust opaque layer were observed in the IR due to a more favorable pointing configuration (type 2). For the three first type 2 occultations, the pointing is satisfactory at IR optical depths smaller than about $0.05(z>15 \mathrm{~km})$. This situation deteriorates at later times due to the progressive change of the geometry. For the last type 2 (S256), the loss of pointing occurs as soon as the Sun is occulted by the dust layer. Contrary to the case of type 1 observations, there is no information on the behavior of the instrument when the loss of pointing occurs for type 2. Nevertheless, comparing the first type 2 , which are not contaminated by pointing problems, to those obtained at the end of the observation period, it may be concluded that the effects of the loss of pointing are less disturbing for type 2 than for type 1 .

The principle of the spectral analysis is the same as in the previous section, except that the light was recorded at only two wavelengths $(1.9$ and $3.7 \mu \mathrm{m})$. Due to the progressive increase of the tangential dust optical thickness when moving downward and because much more than two layers (as was the case for thin clouds) are sounded by the line of sight, the inversion is necessary. It is quite probable that the dust, like clouds, does not present a uniform horizontal distribution and that onedimensional inversion techniques are not adequate. Nevertheless, the eventual inadequacy of the inversion procedure cannot be checked for dust since only two wavelengths, instead of six for clouds, are available and no statistical fit can be made. In the absence of a better alternative, vertical profiles of the tangential optical thickness of the dust at the two IR wavelengths are inverted using the method described in Appendix C. The simplest way to retrieve the effective radius of particles is to compare for each altitude the ratio $\delta \tau(z)=\tau_{1.9}(z) / \tau_{3,7}(z)$ between the extinction coefficients at $1.9 \mu \mathrm{m}$ and $3.7 \mu \mathrm{m}$ to the ratio $\delta \sigma(r)=\sigma_{1.9}(r) / \sigma_{3.7}(r)$ between the theoretical extinction cross-sections at the same wavelengths. The radius $r(z)$ of dust particles at altitude $z$ is the value of $r$ such that $\delta \sigma(r)=\delta r(z)$. Because the noise is weak, the major source of error on the radius is the unknown variance of the size distribution. Calculations of the crosssection are made using real and imaginary indexes of basalt given by Pollack et al. (1973). Using the same synthetic distribution as for water ice [Eq. (1)], the values of $\delta \sigma(r)$ are calculated from 0.1 to $4 \mu \mathrm{m}$ wavelength with a step of $0.1 \mu \mathrm{m}$ for values of $b$ in the range from 0.05 to 0.45 with a step of 0.05 . Viking observations show that Martian dust particles have a large single scattering albedo $(>0.9)$ and a strong forward asymmetry (asymmetry parameter $>0.7$ ). For these particles, extinction is mainly due to scattering by diffraction, which depends on the size and shape but not on the refractive index of the particles (P. Drossart, personal communication). Therefore, the extinction cross-section is not expected to be very sensitive to the choice of a particular refractive index.

Figure 13 shows the curves describing the dependence of $\delta \sigma$ versus the radius for the different values of $b$. The four horizontal straight lines represent the value of $\delta \tau$ at the altitude where the tangential optical thickness at 1.9 $\mu \mathrm{m}$ is 0.1 for the four first type 2 occultations. The segments defined as the intersection of these lines with the beam of the $\delta \sigma(r)$ curves gives the range of the effective radius corresponding to a $b$-range of $0.05-0.45$. The same estimate may be done for each occultation at each altitude. In Fig. 14 is shown for S221 the vertical variation of the effective radius obtained using the previous method. The derived vertical profile of the dust abundance and its scale height are also shown. The characteristics of the dust in the 10 upper kilometers of the dust opaque layer are given in Table II. They are about similar for all occultations: (1) the top of the opaque layer, defined as the altitude where the dust becomes transparent in occultation conditions, is around $25 \mathrm{~km}$ altitude; (2) the particle effective radius is $1 \mu \mathrm{m}$ near the top of the opaque layer, within $30 \%$ accuracy, and increases below at a rate of $\sim 0.05 \mu \mathrm{m}$ $\mathrm{km}^{-1}$; (3) the dust scale height is $3-4 \mathrm{~km}$ below the top of the opaque layer. The similarity of the profiles obtained 


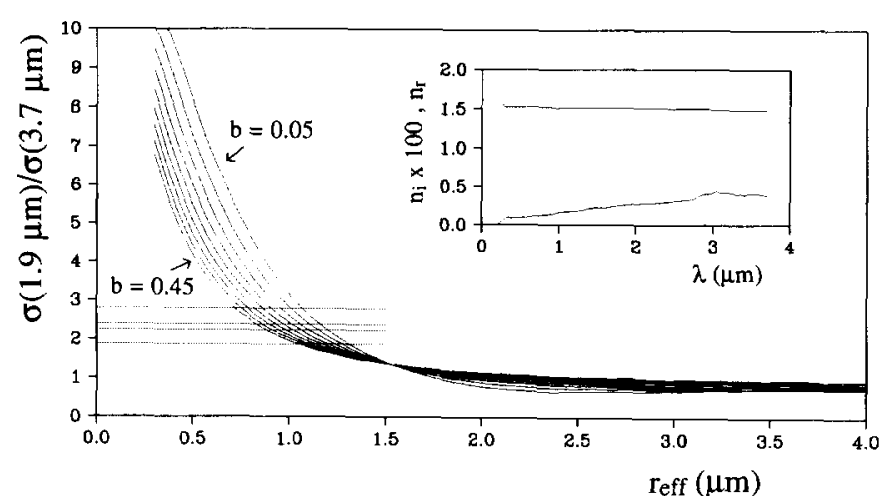

FIG. 13. The ratio between the extinction cross sections of basalt particles at 1.9 and $3.7 \mu \mathrm{m}$ is plotted versus effective radius for nine values of the effective variance from 0.05 to 0.45 . The four horizontal straight lines indicate the values of the ratio between the observed optical depths at 1.9 and $3.7 \mu \mathrm{m}$ for the four first type- 2 occultations at the atmospheric level where the tangential optical thickness at $1.9 \mu \mathrm{m}$ is 0.1 . The segment defined by the intersection of each line with the beam of the theoretical curves provides an estimate of the radius for the corresponding occultation. The inserted figure shows the real and the imaginary parts (resp., upper curve and lower curve divided by 100) of the refractive index, which were chosen for the calculation of cross sections.

at different places and the low vertical extent of the dust opaque layer suggests a quiet and horizontally uniform atmosphere at the seasonal time $\left(L_{S}=0^{\circ}-20^{\circ}\right)$ and latitude $\left(0^{\circ}-20^{\circ} \mathrm{N}\right)$ of the observations. The dust optical depth in the visible cannot be estimated without an extrapolation of the dust profiles below $15 \mathrm{~km}$ altitude down to the
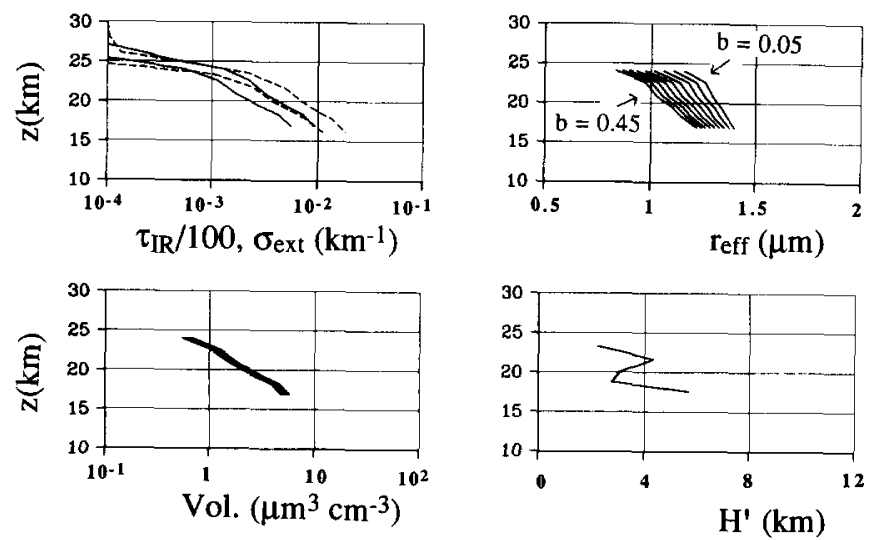

FIG. 14. Left upper part: vertical profiles of the tangential optical thickness divided by 100 at 1.9 and $3.7 \mu \mathrm{m}$ (dashed lines) and of the corresponding extinction coefficient (solid lines). There is less extinction at $3.7 \mu \mathrm{m}$ than at $1.9 \mu \mathrm{m}$. Right upper part: vertical profiles of the effective radius of particles obtained for nine values of the effective variance $b$ in the range $0.05-0.45$. Left lower part: vertical profiles, for the nine values of $b$, of the volumic abundance of particles in units of 1 $\mu \mathrm{m}^{3}$ per cubic centimeter of atmosphere. Right lower part: scale height derived from the profile of the volumic abundance.
TABLE II

Characteristic of the Dust Component at the Top of the Dust Opaque Layer, Defined as the Level where the Tangential Optical Thickness at $1.9 \mu \mathrm{m}$ is Equal to 0.1

\begin{tabular}{|c|c|c|c|c|c|}
\hline & $\begin{array}{c}z_{\text {top }} \\
\text { at } \tau_{1.9}=0.1 \\
(\mathrm{~km})\end{array}$ & $\begin{array}{c}\text { eff. radius } \mathrm{r} \\
\text { at the top } \\
(\mu \mathrm{m})\end{array}$ & $\begin{array}{c}\text {-dr/dz } \\
\left(\mu \mathrm{m} \mathrm{km}^{-1}\right)\end{array}$ & $\begin{array}{c}\text { particle } \\
\text { number density } \\
\left(\mathrm{cm}^{-3)}\right.\end{array}$ & $\begin{array}{c}\text { vol. abund. rat. } \\
\text { scale beight } \\
(\mathrm{km})\end{array}$ \\
\hline 2212158 & $24(+3,-2)$ & $1 \pm 0.2$ & $\approx 0.04$ & $0.3-2$ & $3 \pm 1$ \\
\hline 2360639 & $25(+4,-2)$ & $1 \pm 0.2$ & $\approx 0.05$ & $0.2-1.3$ & $4 \pm 2$ \\
\hline 2362241 & $26(+6,-3)$ & $1.2 \pm 0.1$ & - & $0.2-1.2$ & \\
\hline 2421504 & $27(+2,-2)$ & $0.9 \pm 0.2$ & $\approx 0.05$ & $0.15-1.1$ & $4 \pm 1$ \\
\hline 2440712 & $26(+2,-3)$ & $0.9 \pm 0.2$ & $\approx 0.05$ & $0.25-1.7$ & $4 \pm 1$ \\
\hline 2441514 & $23(+2,-2)$ & $1.1 \pm 0.2$ & $\approx 0.04$ & $0.25-1.8$ & $3 \pm 1$ \\
\hline 2450717 & $23(+2,-2)$ & $0.9 \pm 0.2$ & $\approx 0.06$ & $0.2-1.6$ & $2.5 \pm 0.5$ \\
\hline 2510743 & $28(+4,-2)$ & $0.9 \pm 0.2$ & $\approx 0.05$ & $0.2-1.2$ & $4 \pm 2$ \\
\hline 2560312 & $24(+2,-3)$ & $0.7 \pm 0.2$ & - & $0.15-1.4$ & - \\
\hline
\end{tabular}

Note. The corresponding altitude is indicated in the first column. The effective radius and its gradient (negative because the size increases downward) over one dust scale height below the top of the opaque layer are given in columns 2 and 3 . The scale height of the volumic abundance of dust below the top of the opaque layer and the mean particle number density over one scale height below the top are reported in the last two columns. Due to pointing perturbations, it is sometimes difficult to derive a reliable vertical variation of radius and number density (occultations 2362241 and 2560312).

ground. This study is out of the scope of the present paper but it is in progress and some results have already been presented (Chassefière et al. 1991). A complete study, including comparison with other experiments of the Phobos spacecraft observing the dust, will exist in a near future.

\section{VERTICAL PROFILE OF $K$ DERIVED FROM DUST AND CLOUD OBSERVATIONS}

\section{Analysis of the Dust Component at Low Altitude $(20-25 \mathrm{~km})$}

Assuming for simplicity a monodisperse size distribution, the continuity equation for particles of radius $r$ at the altitude $z$ may be written

$$
K N \frac{d \rho}{d z}+\rho N v_{\mathrm{set}}=0
$$

or

$$
H \frac{d(\log \rho)}{d z}+\frac{V_{\text {set }}}{V_{\text {edd }}}=0,
$$


where $K$ is the eddy diffusion coefficient, $N$ the volumic mass of the ambient atmosphere, $\rho$ the mass mixing ratio of dust particles, $V_{\text {set }}$ the settling velocity of particles of radius $r$ at altitude $z$, and $V_{\text {edd }}$ the eddy mixing velocity. $V_{\text {edd }}$ is defined as the ratio of $K$ to $H$, where $H$ is the atmospheric scale height. The coagulation between particles may be neglected because the characteristic coagulation time $\tau_{\text {coag }}$ is large. Using our values of the number density and the radius of dust particles around $20 \mathrm{~km}$ altitude (respectively, $\sim 1 \mathrm{~cm}^{-3}$ and $1 \mu \mathrm{m}$ ), together with a temperature of $\sim 180 \mathrm{~K}$ and a density of the dust of $2-3$ $\mathrm{g} \mathrm{cm}^{-3}$, a value of $\tau_{\text {coag }}$ of $\sim 10^{8} \mathrm{sec}$ may be inferred. Assuming that $K \approx 10^{6} \mathrm{~cm}^{2} \mathrm{sec}^{-1}$, as shown later, the characteristic times associated with eddy mixing $\left(\tau_{\text {edd }} \approx\right.$ $\left.\mathrm{H}^{2} / K\right)$ and settling $\left(\tau_{\mathrm{set}} \approx H / V_{\text {set }}\right)$ are of the order of $10^{6}$ sec, i.e., much lower than $\tau_{\text {coag }}$. Equation (2), whose time constant $\tau_{\mathrm{d}} \approx \max \left(\tau_{\text {edd }}, \tau_{\text {set }}\right) \approx 1$ week, expresses the balance between the upward eddy flux and the downward settling flux in the steady state. Observations, which extend over a time period of 1 month $\left(>\tau_{\mathrm{d}}\right)$, show no significant variations of the dust vertical profile and of its size distribution. Therefore, the steady state hypothesis may be considered as acceptable in first approximation. Eventual diurnal variations of the dust, which cannot be detected since the atmosphere is observed at sunset by the solar occultation technique, are expected to have little effect since they act on a time period definitely smaller than $\tau_{\mathrm{d}}$. Denoting by $H^{\prime}(z)$ the local scale height of the dust at altitude $z$, in such a way that

$$
\rho N=\rho_{0} N_{0} \exp \left(-\frac{z-z_{0}}{H^{\prime}}\right)
$$

or

$$
\rho=\rho_{0} \exp \left(-\left(\frac{1}{H^{\prime}}-\frac{1}{H}\right)\left(z-z_{0}\right)\right) .
$$

Equation (3) may be rewritten as

$$
H^{\prime}=\frac{H}{1+\frac{V_{\mathrm{set}}}{V_{\mathrm{edd}}}}=\frac{H}{1+R_{\mathrm{v}}},
$$

where $R_{\mathrm{v}}$ is the ratio of the settling velocity to the eddy diffusion velocity. As shown in Table II, the local scale height of dust around $20 \mathrm{~km}$ altitude is in the range from 2 to $6 \mathrm{~km}$, with an average value of about $3.5 \mathrm{~km}$. Assuming an atmospheric temperature at this altitude of 180-220 $\mathrm{K}$ with a mean value of $200 \mathrm{~K}$ (Krasnopolsky, 1986), i.e., an atmospheric scale height of $10 \pm 1 \mathrm{~km}$, Eq. (6) yields $R_{\mathrm{v}} \approx 0.5-5$ (taking into account the uncertainty on $H^{\prime}$ ) with a most likely value of $\sim 2$ around $20-25 \mathrm{~km}$ altitude. Because the radius of particles is much smaller than the mean molecular free path $(\sim 100 \mu \mathrm{m}$ at the ground level), the fall velocity of particles varies as $r$. We will use in the present study the values of the fall velocity given by Michelangeli et al. (1992) for ice crystals. Values for dust particles are multiplied by a factor 2-3 due to their larger density. For particles of radius $1 \mu \mathrm{m}$ at $20 \mathrm{~km}$ altitude, a fall velocity of $\sim 2-3 \mathrm{~cm} \mathrm{sec}^{-1}$ is inferred. A value of $R_{\mathrm{v}}=1.5-2$ yields an eddy diffusion velocity of $\sim 1 \mathrm{~cm}$ $\mathrm{sec}^{-1}$, i.e., $K \approx 10^{6} \mathrm{~cm}^{2} \mathrm{sec}^{-1}$ within about a factor of 3 on both sides. A detailed calculation was performed for each type 2 occultation and precise results, cumulating all sources of uncertainty, will be presented together with those obtained for clouds in the next section.

The theoretical variations of effective radius and variance as a function of altitude may be derived from Eqs. (1) and (3). Since $V_{\text {set }}$ is proportional to the radius $r$ at any altitude $\left(V_{\text {set }}=r \overline{V_{\text {set }}}\right)$, the mixing ratio $\rho$ of particles of radius $r$ at altitude $z$ may be deduced from $\rho_{0}$ at altitude $z_{0}$ using Eq. (3),

$$
\rho=\rho_{0} \exp \left(-r \frac{1}{H} \int_{Z_{0}}^{7} \overline{V_{\text {edd }}} d z\right)
$$

It is justified to treat each radius range of the particle size distribution independently of each other since the motion of particles is driven by the fluid atmosphere, not by the collisions with other particles. Indeed, the characteristic time of the frictional coupling between a particle and the surrounding atmosphere is very short $(<<1 \mathrm{sec})$. From Eqs. (1) and (7), it may be deduced that there is no change of the effective variance $b$ between $z_{0}$ and $z$ and the effective radius $a$ at altitude $z$ is given by

$$
\frac{1}{a b}=\frac{1}{a_{0} b}+\frac{1}{H} \int_{z_{01}}^{z} \overline{V_{\text {set }}} d z .
$$

For a small $\Delta z=z-z_{0}$, Eq. (8) may be differentiated as follows:

$$
H \frac{d \ln (\mathrm{a})}{d z} \approx-b \frac{V_{\mathrm{set}}}{V_{\mathrm{edd}}}
$$

where $V_{\mathrm{set}}$ is the settling velocity or particles of radius $a$, which varies inversely as the atmospheric density. At low altitude, where the atmospheric density is large and $V_{\text {set }}$ is consequently small, a little depends on $z$. At high altitude, the equivalent scale height $h\left(h=(d \ln (\mathrm{a}) / d z)^{-1}\right)$ of the effective radius decreases and becomes proportional to the atmospheric density. Vertical profiles of $a$ and $b$ derived from the present analysis are qualitatively in good 
agreement with exact calculations performed by Michelangeli et al. (1992). From Eq. (9), the effective variance, constant with altitude, may be expressed as a function of $h$ as

$$
b=\frac{H}{h} R_{\vee}^{-1}
$$

Since nine vertical profiles of the effective radius below the top of the dust opaque layer are obtained, the effective variance $b$ may be estimated from Eq. (10). $h$ is $\sim 25-35$ $\mathrm{km}$ around $20 \mathrm{~km}$ altitude (Fig. 13; Table II), yielding a ratio $H / h$ of $\sim 1 / 3$. Taking $R_{v} \approx 1.5$, the preferred value of $b$ is $\sim 0.25( \pm 50 \%)$.

\section{Analysis of the Cloud Component at High Altitude $(45-60 \mathrm{~km})$}

We use the cloud model presented in PSP, which was taken from Krasnopolsky (1986). In the same way as for dust in the low atmosphere, the balance equation for water may be written

$$
K\left(\frac{d n_{\mathrm{g}}+d n_{\mathrm{s}}}{d z}+\frac{n_{\mathrm{g}}+n_{\mathrm{s}}}{H}\right)+n_{\mathrm{s}} V_{\mathrm{set}}=0
$$

where $n_{\mathrm{g}}$ is the density of molecules in the gaseous phase, given by the saturation pressure law

$$
n_{\mathrm{g}}=2.210^{12} \exp \left(\frac{T-180}{\alpha}\right) \mathrm{cm}^{-3}
$$

where $\alpha=5.2 \mathrm{~K}$ and $n_{\mathrm{s}}$ is the density of molecules in the solid phase. Equation (11) expresses the balance between the upward eddy flux of water (vapor and ice) and the downward settling flux of water ice. Assuming $K, V_{\text {set }}$, and $\gamma$ (the temperature gradient) constant over the height of the cloud, Eq. (11) can be solved and the solution is (Krasnopolsky 1986)

$$
\begin{aligned}
n_{\mathrm{s}}=c n_{\mathrm{g}_{0}}\left[\exp \left(\frac{\gamma}{\alpha}\left(z-z_{0}\right)\right)\right. & \\
& \left.-\exp \left(-\left(\frac{V}{K}+\frac{1}{H}\right)\left(z-z_{0}\right)\right)\right],
\end{aligned}
$$

where $c$ is a constant,

$$
c=\frac{\frac{\gamma}{\alpha}-\frac{1}{H}}{\frac{1}{H}+\frac{V}{K}-\frac{\gamma}{\alpha}},
$$

and $n_{\mathrm{g}_{0}}$ is the water vapor density at the base of the cloud $\left(z=z_{0}\right)$, where the water partial vapor pressure is equal to its saturation value at the temperature $T\left(z_{0}\right)$. This equation was given with a printing error in PSP (Eq. (5) in PSP) but the correct formula was used for calculations. The average width at half maximum of the vertical extinction coefficient profiles of the clouds observed from Phobos is $\sim 3-6 \mathrm{~km}$ (see Table I), which corresponds to about onehalf atmospheric scale height. Because of the sharpness of the lower and upper edges of the observed clouds, we can assume that this width, derived from an optical profile, is also the width of the physical object. To assume $K$ and $\gamma$ constant in the cloud is therefore justified. The settling velocity is proportional to the ratio of the particle radius to the atmospheric density. The observed increase of the particle radius from the top to the peak extinction layer is about $50 \%$ on average ( $\sim 80 \%$ for $\mathrm{C} 236, \sim 10 \%$ for $\mathrm{C} 251$, see Table I), i.e., of the same order of magnitude as the increase of the atmospheric density over one-half atmospheric scale height. Therefore, in a first approximation, the settling velocity can be considered as constant inside these thin clouds.

The steady state approximation requires mainly that the atmospheric temperature at the cloud level has no diurnal variation and that replenishment of water vapor and condensation nuclei from below is ensured by eddy mixing. These assumptions will be discussed and justified in the last section. Because we are aware of the difficulties inherent to the steady state model, we will define $K_{\text {eq }}$ as the value of the eddy diffusion coefficient $K$ which would produce the observed cloud under steady state conditions. If there is no diurnal variation of the temperature, $K_{\text {eq }}=$ $K$.

The solution given by Eq. (13) has some noteworthy properties. For large values of $K_{\text {eq }}$, Eq. (13) becomes

$$
n_{\mathrm{s}}=n_{\mathrm{g}_{0}}\left[\exp \left(-\frac{1}{H}\left(z-z_{0}\right)\right)-\exp \left(-\frac{\gamma}{\alpha}\left(z-z_{0}\right)\right)\right],
$$

implying that the width of the cloud tends toward a finite value, not much larger than the atmospheric scale height $H$, which is also the scale height of the cloud in its upper part. For small values of $K_{\text {eq }}$, Eq. (13) may be written

$$
\begin{aligned}
n_{\mathrm{s}}= & n_{\mathrm{g}_{0}} \frac{K_{\mathrm{eq}}}{V_{\mathrm{set}}}\left(\frac{\gamma}{\alpha}-\frac{1}{H}\right) \\
& {\left[\exp \left(-\frac{\gamma}{\alpha}\left(z-z_{0}\right)\right)-\exp \left(-\frac{V_{\text {set }}}{K_{\mathrm{eq}}}\left(z-z_{0}\right)\right)\right], }
\end{aligned}
$$

which may be approximated, at the level immediately above $z_{0}$, by 


$$
n_{\mathrm{s}}=n_{\mathrm{g}_{0}} \frac{K_{\mathrm{eq}}}{V_{\mathrm{set}}}\left(\frac{\gamma}{\alpha}-\frac{1}{H}\right) \exp \left(-\frac{\gamma}{\alpha}\left(z-z_{0}\right)\right)
$$

The scale height of the cloud is in this case $\alpha / \gamma(2-3$ $\mathrm{km})$. In a first approximation, the width of the cloud $W$ is near $\alpha / \gamma$ for values of $V_{\text {edd }}\left(=K_{\text {eq }} / H\right)$ much smaller than $V_{\text {set. }}$. For increasing $K_{\text {eq }}, W$ increases and tends toward an upper limit $(\sim H)$ for values of $V_{\text {edd }}$ much larger than $V_{\text {set }}$. For a fixed peak value of $n_{\mathrm{s}}$ at a given level, the water vapor density $n_{\mathrm{g}_{0}}$ at the base of the cloud does not depend on $K_{\text {eq }}$ for large values of $K_{\mathrm{eq}}$, and is inversely proportional to $K_{\text {eq }}$ for small values of $K_{\text {eq }}$.

The parameters of the model are $T_{0}$ [or $\rho_{\mathrm{wp}}$, the water vapor mixing ratio linked to $T_{0}$ by Eq. (12)], influencing the particle number density, the temperature gradient, $\gamma$, and the equivalent eddy diffusion coefficient $K_{\text {eq }}$, both influencing the width of the cloud. The exploration of the space of parameters has been made as described now. Four possible values of the gradient (negative) were tested: $1,1.5,2$, and $2.5 \mathrm{~K} \mathrm{~km}^{-1}$. This choice is motivated by the following considerations. The typical expected gradient at this altitude is $1 \mathrm{~K} \mathrm{~km}^{-1}$. As shown hereafter, this value is definitely too low to fit observations and it is not necessary to explore lower values. A gradient of 2.5 $\mathrm{K} \mathrm{km}^{-1}$ is high enough to fit our thinnest cloud and because a still higher gradient is implausible, it is not useful to explore larger values. A relatively large step of $0.5 \mathrm{~K}$ between $1 \mathrm{~K} \mathrm{~km}^{-1}$ and $2.5 \mathrm{~K} \mathrm{~km}^{-1}$ was chosen in order to minimize the computation time and simplify the presentation of results. For each one of these four values of the thermal gradient, cloud profiles have been generated for a large number of values of $K_{\text {eq }}$ varying from $10^{4}$ to $10^{9}$ $\mathrm{cm}^{2} \mathrm{sec}^{-1}$. In each case, an iterative procedure allows adjustment of altitude and resolution to those of the observed cloud and allows the value of $T_{0}$ (and $\rho_{\mathrm{wp}}$ ) to be inferred from the observed value of the water ice molecule density at the peak extinction level. In Figs. 15 to 17 are shown for the four values of $\gamma$ the best fits of the calculated ice profiles to the observed profiles (for resp. C231, C236, and C251) when exploring the complete range of $K_{\text {eq }}$. For C231 (Fig. 15), it is not possible to fit the cloud with a small value of $\gamma$, for which the lower limit of the cloud width $\left(\sim \alpha / \gamma\right.$ when $\left.K_{\mathrm{eq}} \rightarrow 0\right)$ is still larger than the observed width. Increasing $\gamma$ to $2.5 \mathrm{~K} \mathrm{~km}^{-1}$, it becomes possible to produce a cloud as thin as the one observed. In Fig. 15 is shown for C231 the variation of the ratio between the calculated half-width and the corresponding observed quantity as a function of $K_{\mathrm{eq}}$. This is a band because we retain three possible values of $\gamma\left(1.5,2\right.$, and $\left.2.5 \mathrm{~K} \mathrm{~km}^{-1}\right)$, rejecting the lowest one $\left(1 \mathrm{~K} \mathrm{~km}^{-1}\right)$, which is in obvious contradiction with data. An upper limit of $K_{\text {eq }}$ may be inferred $\left(2 \times 10^{5} \mathrm{~cm}^{2} \mathrm{sec}^{-1}\right)$. The corresponding variations of $T_{0}$ and $\rho_{\mathrm{wp}}$ as a function of $K_{\mathrm{eq}}$ are also plotted. Ac-
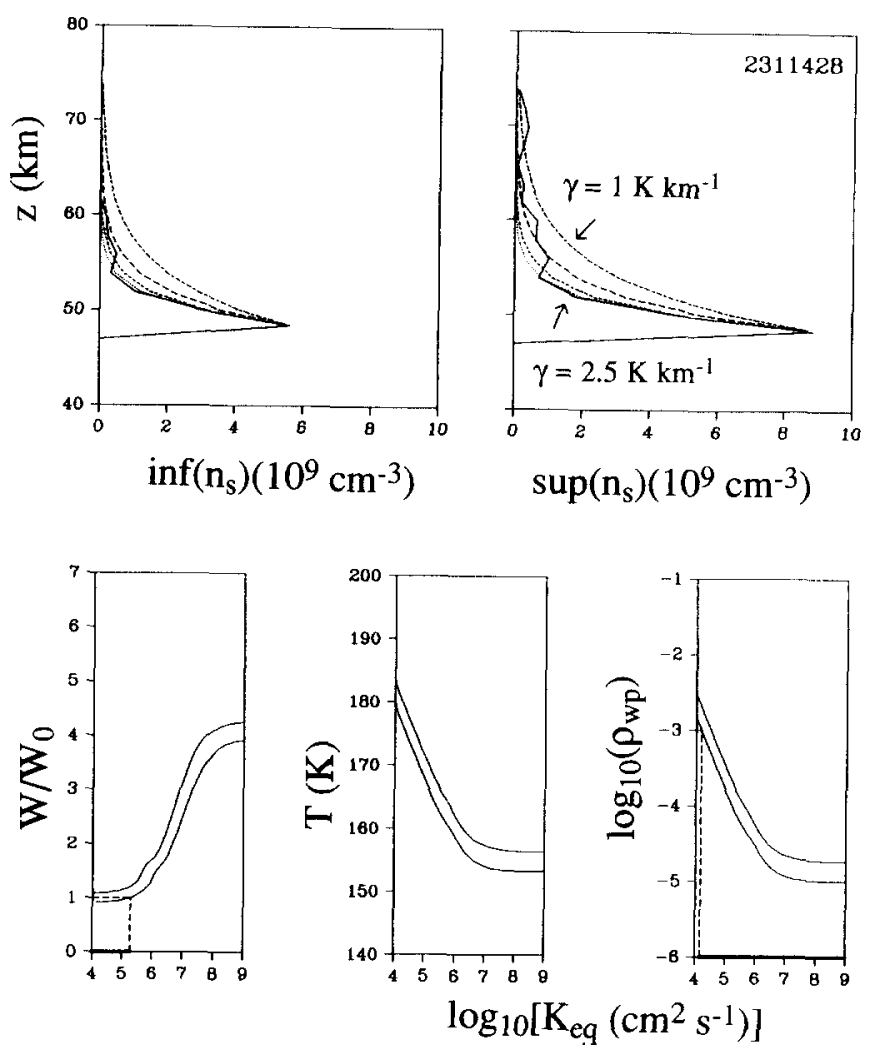

FIG. 15. In the upper part are plotted with a solid line the vertical profiles of the upper (right side) and lower (left side) limit on the number density of water ice molecules in units of $10^{9} \mathrm{~cm}^{-3}$ for $\mathrm{C} 231$. The four profiles plotted in each figure with different kinds of dashed lines are those giving the best fit of model to data for $\gamma=1$ (wide cloud), 1.5, 2, and $2.5 \mathrm{~K} \mathrm{~km}^{-1}$ (narrow cloud), $K_{\text {eq }}$ varying from $10^{4}$ to $10^{9} \mathrm{~cm}^{2} \mathrm{sec}^{-1}$. In the three lower figures are represented from the left to the right: (1) The ratio of the width at half maximum of the calculated cloud profile to the observed one as a function of $K_{\text {eq }}$. This is a band because several values of the temperature gradient are retained $\left(1.5\right.$ to $\left.2.5 \mathrm{~K} \mathrm{~km}^{-1}\right)$. The range of the compatible values of $K_{\mathrm{eq}}$ is indicated by a thick segment on the $x$-axis. (2) The corresponding variation of the temperature. (3) The corresponding variation of the water vapor mixing ratio. The range of $K_{\text {eq }}$ compatible with a mixing ratio smaller than $10^{-3}$ is indicated by a thick segment on the $x$-axis.

cording to the general properties of the solution previously mentioned, there is for small values of $K_{\text {eq }}$ an inverse proportionality between $\rho_{\text {wp }}$ and $K_{\text {eq }}$, which may be seen also on temperature plots. Assuming that the water vapor mixing ratio at this time and latitude cannot exceed an upper limit of $10^{-3}$, which corresponds to a vertical column abundance of 50 pr. $\mu \mathrm{m}$, a lower limit on $K_{\text {eq }}\left(\sim 10^{4}\right.$ $\mathrm{cm}^{2} \mathrm{sec}^{-1}$ ) may be deduced. An excellent fit is obtained for $\mathrm{C} 236$ (Fig. 16). As previously, a thermal small gradient of $1 \mathrm{~K} \mathrm{~km}^{-1}$ may be rejected. The constraint on the width yields an upper limit on $K_{\text {eq }}$ of $\sim 510^{6} \mathrm{~cm}^{2} \mathrm{sec}^{-1}$, the constraint on $\rho_{\mathrm{wp}}$, providing a lower limit of $2 \times 10^{5}$ $\mathrm{cm}^{2} \mathrm{sec}^{-1}$. C251 (Fig. 17) is the only case for which an 

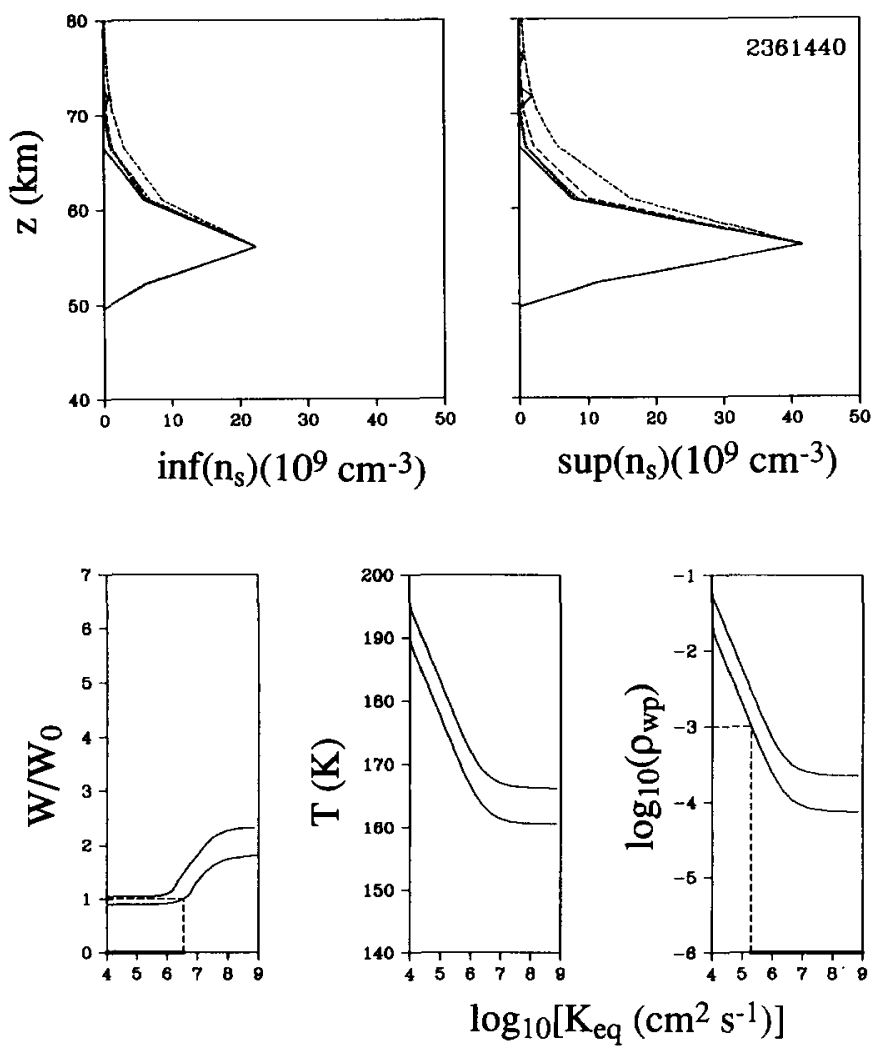

FIG. 16. Same as Fig. 15 for C236.

adjustment of the width allows both an upper $\left(\sim 5 \times 10^{6}\right.$ $\left.\mathrm{cm}^{2} \mathrm{sec}^{-1}\right)$ and a lower $\left(\sim 5 \times 10^{5} \mathrm{~cm}^{2} \mathrm{sec}^{-1}\right)$ limit on $K_{\text {eq }}$ to be defined. In all cases, the temperature gradient must be larger than $1.5 \mathrm{~K} \mathrm{~km}^{-1}$, with a preferred value of $\sim 2$ $\mathrm{K} \mathrm{km}^{-1}$.

In Fig. 18 are summarized results obtained from both dust and clouds. On the left side are represented the domain of the possible values of $K_{\mathrm{eq}}$ for each one of the four observed clouds, derived from the previous analysis. Thick solid lines give upper limits derived from the constraint on the width; they depend on the altitude because of its influence on the settling velocity of particles, which is inversely proportional to the atmospheric density. In the case of C251, the field may be closed on the left because a lower limit on $K_{\text {eq }}$ may be derived too (oblique thin solid line). In other cases, a lower limit is obtained assuming that the water vapor mixing ratio is lower than $10^{-3}$ (oblique dashed lines). Values of $K_{\mathrm{eq}}$ corresponding to a water vapor mixing ratio of $2 \times 10^{-4}(10 \mathrm{pr} . \mu \mathrm{m}$, as observed by the Viking MAWD experiment) are indicated inside each field by a thin dotted line. Based on the analysis of the width, an upper limit of $2 \times 10^{5}-5 \times 10^{6} \mathrm{~cm}^{2}$ $\mathrm{sec}^{-1}$ on $K_{\text {eq }}$ may be inferred assuming that clouds are all located between 50 and $55 \mathrm{~km}$. Corresponding values of the temperature are indicated in the center part of the
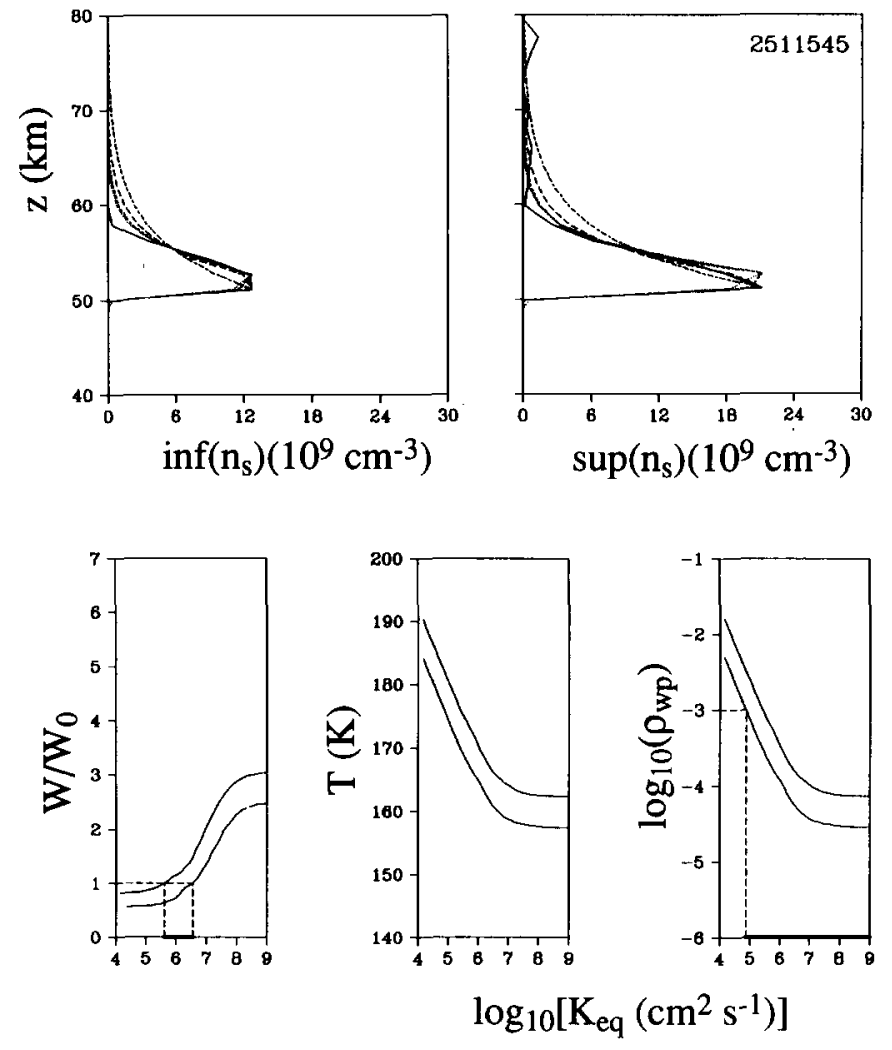

FIG. 17. Same as Fig. 15 for C251.
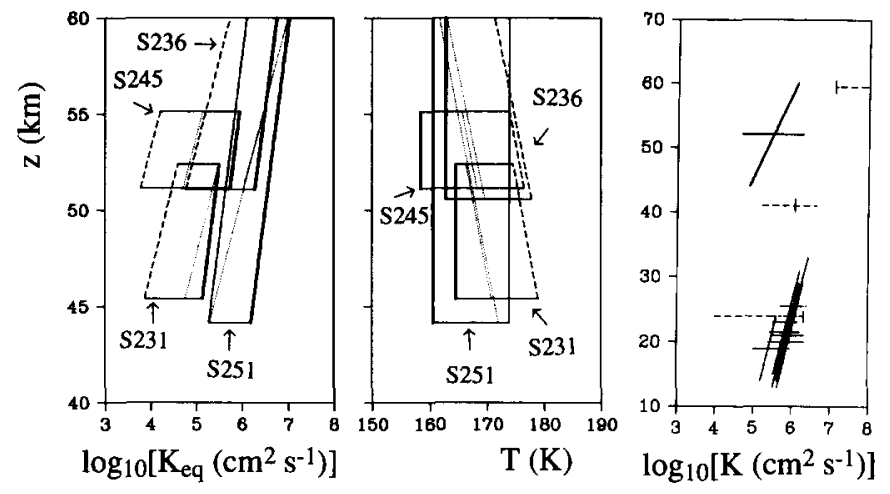

FIG. 18. On the left are shown the values of $K_{\mathrm{eq}}$ derived from the analysis of the four observed clouds. Thick vertical oblique lines represent upper limits based on the analysis of the width. The thin vertical oblique line represents a lower limit based on the analysis of the width for $\mathbf{S} 251$. Dashed lines represent lower limits derived from the constraint on the water vapor $\left(\rho_{\mathrm{wp}}<10^{-3}\right)$ and thin dotted lines correspond to $\rho_{\mathrm{wp}}=2 \times 10^{-4}$. In the middle are shown corresponding values of the temperature with the same definitions. On the right are plotted the values of $K$ derived from the dust for six type 2 occultations (20-25 km altitude) and a synthetic value of $K_{\text {eq }}$ obtained putting together results obtained for the four observed clouds (50-55 km altitude). Values deduced from the analysis of the three Viking clouds are indicated by dashed lines. Vertical segments intersecting these dashed lines correspond to values of the effective radius adopted by Kahn (1990). 

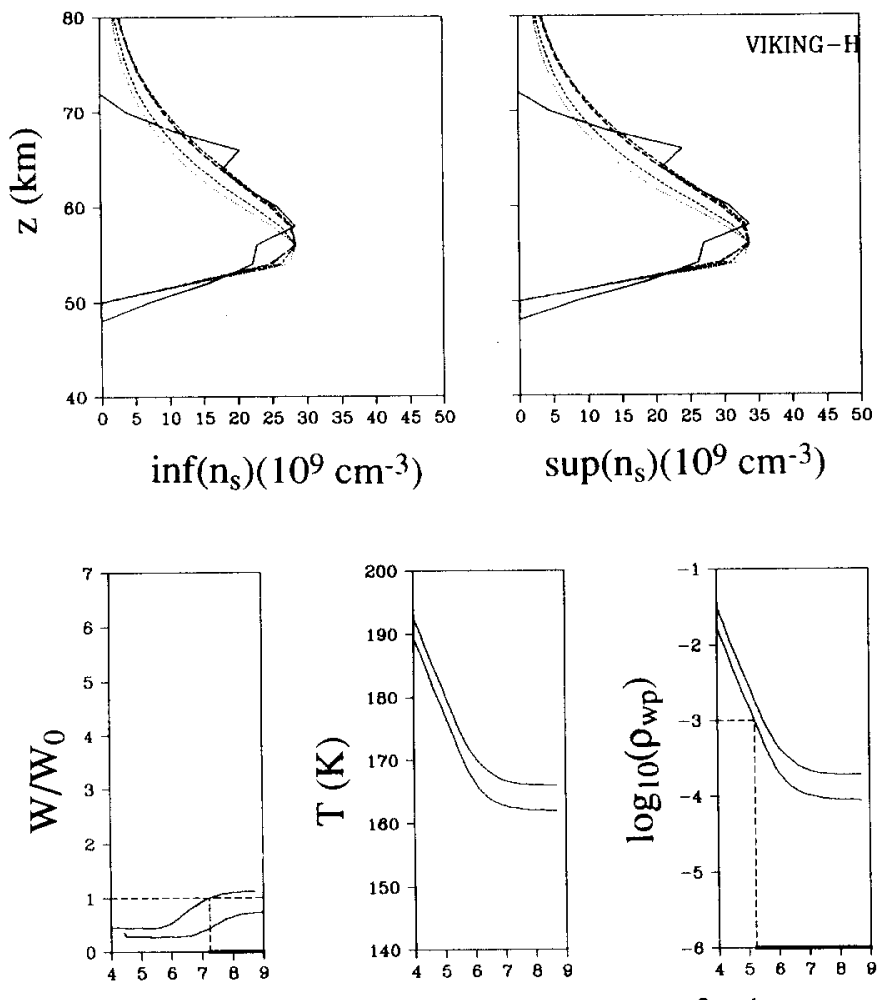

$\log _{10}\left[\mathrm{~K}\left(\mathrm{~cm}^{2} \mathrm{~s}^{-1}\right)\right]$

FIG. 19. Same as Fig. 15 for the highest Viking cloud H.

figure. The lower limit of the temperature at the base of the cloud is $160-165 \mathrm{~K}$ with a most probable value of 170 $\mathrm{K}$. On the right side are shown together the values of $K$ derived from the dust for type 2 occultations and a synthetic value of $K_{\mathrm{eq}}$, summarizing the values obtained for individual clouds. Both $K$ at low altitude and $K_{\text {eq }}$ at high altitude are betwen $\sim 10^{5}$ and $\sim 310^{6} \mathrm{~cm}^{2} \mathrm{sec}^{-1}$ with an average value just below $10^{6} \mathrm{~cm}^{2} \mathrm{sec}^{-1}$.

\section{Analysis of Three Clouds Observed by the Viking Orbiter}

Some clouds were also observed in the Viking Orbiter images and the above-mentioned simplified treatment has been applied to the three clouds recently analyzed by Kahn (1990, 1991). Vertical extinction profiles of these clouds, detected at midlatitudes in summer and early winter viewing at the limb of Mars and denoted, respectively, by $\mathrm{L}$ (low, $z \approx 20 \mathrm{~km} ; L_{\mathrm{S}}=338^{\circ}$ ), $\mathrm{M}$ (middle, $z \approx 36 \mathrm{~km}$; $\left.L_{\mathrm{S}}=107^{\circ}\right)$, and $\mathrm{H}\left(\right.$ high, $\left.z \approx 50 \mathrm{~km} ; L_{\mathrm{S}}=139^{\circ}\right)$ are obtained less directly than for solar occultation since additional information on optical and morphological properties of particles are required. In Fig. 19 are shown the vertical profiles of the lower and upper limits on the number density of water ice molecules for the highest Viking cloud $(\mathrm{H})$, assuming that the effective radius of particles, which is not known, is in the range from 0.2 to $1 \mu \mathrm{m}$. These densities are obtained by dividing the extinction coefficients, as calculated by Jaquin et al. (1986), by our extinction cross-sections and their reliability might be questioned for the following reason. In principle, simulations should be compared to the observed quantity, namely the product of the single scattering albedo, phase function, and extinction cross section. Such an approach would complicate the analysis and in view of its aim, the estimate of $K_{\text {eq }}$, would not change anything since $K_{\text {eq }}$ depends on the shape of the profile and not on the absolute extinction. Because the particle radius does not change much over the cloud, as deduced from the analysis of Phobos clouds, the vertical profiles of the scattered light and of the number density, as shown in Fig. 19, are expected to be nearly proportional. $K_{\mathrm{eq}}$ also depends on the radius of the particles through their settling velocity but the large uncertainty assumed for the effective radius may be assumed to take into account eventual errors due to a bad choice of simple scattering albedo and phase function.

Theoretical profiles corresponding to the best fits are shown in Fig. 19 for the four possible values of the temperature gradient, from 1 to $2.5 \mathrm{~K} \mathrm{~km}^{-1}$. Contrary to the case of the very thin Phobos clouds, no information on the temperature gradient can be deduced. This is also true for the two other Viking clouds. For the lowest cloud L, the allowed range of $K_{\mathrm{eq}}$ values is rather similar to results obtained from the Phobos clouds $\left(K_{\mathrm{eq}}<10^{6} \mathrm{~cm}^{2} \mathrm{sec}^{-1}\right)$. The thicker intermediate cloud $\mathbf{M}$ cannot be reproduced for too small a value of $K_{\mathrm{eq}}\left(10^{5} \mathrm{~cm}^{2} \mathrm{sec}^{-1}<K_{\mathrm{eq}}<10^{7}\right.$ $\left.\mathrm{cm}^{2} \mathrm{sec}^{-1}\right)$. Due to the large width of the highest cloud $\mathrm{H}$ (Fig. 19), observed in late southern summer at $-37^{\circ}$ latitude, a high eddy mixing rate is required $\left(K_{\text {eq }}>10^{7}\right.$ $\left.\mathrm{cm}^{2} \mathrm{sec}^{-1}\right)$. No upper limit may be inferred due to the fact that even for very large values of $\mathbf{K}_{\mathrm{eq}}$, it is not possible to generate clouds having a vertical extent much larger than one atmospheric scale height. The disagreement between our results and those presented by Kahn in his first analysis (Kahn 1990) is now understood and it is due to an error in the calculation of Kahn. Our estimates of $K_{\mathrm{eq}}$ are consistent with the revised estimates of Kahn (1991). The values of $K_{\text {eq }}$ obtained for the three Viking clouds from the present analysis are shown in Fig. 18. The dashed horizontal segment indicates the range obtained assuming an effective radius of $0.6 \pm 0.4 \mu \mathrm{m}$. The vertical segment indicates the value of $K_{\mathrm{eq}}$ corresponding to the radius estimated by Kahn $(6,0.7$, and $0.15 \mu \mathrm{m}$ for, resp., L, M, and $\mathrm{H}$ cloud). For the lowest cloud, the dashed line $\left(r_{\text {eff }}=0.2-1 \mu \mathrm{m}\right)$ has been extended up to $r_{\text {eff }}=6 \mu \mathrm{m}$ (dotted line). The increase of $K_{\mathrm{eq}}$ above $50 \mathrm{~km}$ is not surprising in view of high values obtained from Viking mass spectrometer measurements at high altitude ( $K=$ $2-5 \times 10^{7} \mathrm{~cm}^{2} \mathrm{sec}^{-1}$ at $100 \mathrm{~km}$ altitude) . 


\section{DISCUSSION}

\section{Justification of the Steady State Model}

Before we discuss implications of the above results, we return to the basic question as to whether our treatment of clouds using a steady state approximation is adequate. Assuming for the clouds an average width at half maximum, $W$, of $\sim 4 \mathrm{~km}$ together with an eddy diffusion coefficient $K$ of $10^{6} \mathrm{~cm}^{2} \mathrm{sec}^{-1}$, the time constant for eddy diffusion through the cloud $\left(W^{2} / K\right)$ is about 1 Martian day. For an ice particle of radius $0.5 \mu \mathrm{m}$ at $50 \mathrm{~km}$, the fall velocity is $\sim 20 \mathrm{~cm} \mathrm{sec}-1$ (Michelangeli et al. 1992), implying a mean time to fall $4 \mathrm{~km}$ of $\sim 6 \mathrm{hr}$. In the presence of a diurnal fluctuation of the temperature, the state of the cloud at a given time is therefore not correctly described by the steady state model. A clear signature of diurnal variability of optical depth was found in Viking lander optical depth data by Colburn et al. (1989) and attributed by them to water ice cloud formation and dissipation. These authors plot on their Figs. 15 and 16 the difference between AM and PM optical depths as a function of the seasonal date. It is interesting to note that only small diurnal variations were detected at lander $1\left(22^{\circ} \mathrm{N}\right)$ near $L_{\mathrm{S}}=0$, the approximate seasonal date of the Phobos observations, although a small shift of the peak at $L_{\mathrm{S}} \approx$ $320^{\circ}$ toward the northern spring equinox at the beginning of 1989 could lead to the opposite conclusion. In all cases, it appears that there are times and places where it is reasonable to neglect diurnal variations, but there are no general justifications for such a neglect. The aim of the present discussion is to show why diurnal variations are expected to be small at the time of Phobos observations and therefore can be reasonably neglected. There are several arguments in favor of the steady state assumption used here:

(1) During the period of observations, made at low latitude and northern spring equinox, the atmosphere is transparent above $25 \mathrm{~km}$ altitude, the scale height of the dust around $20-25 \mathrm{~km}$ being $\sim 3.5 \mathrm{~km}$. The inversion of cloud profiles shows that the atmosphere is clear below the base of the cloud (Figs. 8 to 11), although some opacity could exist rather high in the atmosphere, as suggested by infrared measurements during S231 (Fig. 1). In one case (C245), the atmosphere is perfectly clear over $\sim 30 \mathrm{~km}$ below the cloud. The scarcity of dust at the cloud level suggests that the diurnal fluctuation of temperature at 40-60 km altitude is mainly driven by the radiative cooling in the $15-\mu \mathrm{m} \mathrm{CO}_{2}$ band, with a cooling rate of $\sim 10 \mathrm{~K} /$ day and therefore a difference between AM and PM temperatures not in excess of $\sim 5 \mathrm{~K}$.

(2) Clouds are detected at sunset by definition of the solar occultation technique. Figure 10a of the paper by Michelangeli et al. (1992) shows that clouds disappear during late morning and the afternoon, with a depletion by at least two orders of magnitude with respect to the morning maximum. A nearly complete disappearance of condensed water between 2 and 8 PM is also predicted by Colburn et al. (1989) as seen on their Fig. 17 with a contrast by a factor $\sim 50$ between the morning maximum and the evening minimum. Because the eddy mixing time is at least 10 days at the cloud level, clouds are not expected to change altitude during the diurnal cycle. The absolute amount of ice, and therefore also its mixing ratio since altitude does not change, must be multiplied by a factor of 50-100 when extrapolating back the cloud vertical structure at sunset to morning conditions. Since a water vapor mixing ratio of at least $\sim 10^{-4}$ is required to explain the clouds observed from Phobos in the evening, the same analysis would lead to a minimum mixing ratio of $5 \times 10^{-3}$ under morning conditions. Such a high value is clearly unacceptable. The observations of the ISM instrument show that the average $\mathrm{H}_{2} \mathrm{O}$ mixing ratio is $\sim 3 \times 10^{-4}$ (Rosenquist et al. 1992), of the same order as the value deduced from our analysis of the clouds. More precisely, Phobos results are incompatible with a contrast between morning and evening quantities of water ice at $-50 \mathrm{~km}$ altitude larger than $\sim 3$. The fact that clouds are observed at $6 \mathrm{PM}$, when they should not exist in the presence of a diurnal temperature variation, seems therefore to support our first argument, that is the diurnal fluctuation of temperature at $\sim 50 \mathrm{~km}$ altitude is weak. The two lowest Viking clouds were observed in late morning and midafternoon (11.10 and $16.40 \mathrm{LT})$. At these times, models predict that clouds have already partly or totally disappeared. The fact that $K$ is in the same range for clouds observed at $11.10,16.40$, and $18.00 \mathrm{LT}$, outside the predicted period of appearance, is a strong argument in favor of the steady state model.

(3) Figure 10 of the paper by Michelangeli et al. (1992) shows also that the width of the cloud does not change significantly during the very short evaporation phase. Therefore, even if some evaporation occurs prior to the time of measurements, results concerning $K$ could be only slightly affected.

(4) Clouds were detected 6 of 38 times, that is in $15 \%$ of cases. This does not correspond to the conditions of a 1-D model. Because of the patchy coverage, replenishment of dust and water vapor at high altitudes is less problematic than in the model of Michelangeli et al. (1992). Due to zonal winds, the region of the atmosphere above the cloud is expected to continually change due to gradients of horizontal velocity. Replenishment must occur through eddy transport in the regions where clouds are not present, and subsequently be more efficient than in the 1-D model.

For these reasons, we feel that a preliminary analysis using the steady state assumption, particularly well adapted to thin patchy clouds in a clear atmosphere, may 
help modelers of Martian clouds. The advantage of the steady state model is that the code is not time consuming and therefore a great number of profiles can easily be generated. As shown above, an excellent agreement between the data and the model is obtained. The present study should be considered as preliminary, as a step toward a more refined modeling. The values of the parameters derived from the present work, namely the temperature, the temperature gradient, and the eddy diffusion coefficient, may be included in a time-dependent model in order to evaluate the exact impact of the diurnal cycle on the structure and the opacity of the cloud.

The good agreement between values obtained from clouds and those, less model dependent, derived from dust at low altitude also supports the model. Because of the strong similarity of dust profiles, the atmosphere is expected to be quiet, basically nonconvective, with a low eddy mixing rate whose diurnal average is expected to be nearly constant in space and time in the short period of Phobos observations. As already mentioned, dust particles fall only 2 to $3 \mathrm{~km}$ per day at $20-25 \mathrm{~km}$ altitude and the vertical structure of dust does not follow eventual diurnal variations of atmospheric characteristics. For these reasons, the value of $K$ derived from the analysis of the dust seems to be reliable and the good coincidence between it and the value deduced from clouds reinforces the plausibility of the steady state model.

\section{Possible Role of the General Circulation}

The eddy diffusion coefficient incorporates variable processes, wave activity, thermal gradients, interacting with the topography. In general, the use of a single coefficient linking the gradient of the mixing ratio to the heat or mass fluxes by a proportionality relationship is somewhat schematic and may be questionable. It assumes in particular that the spatial scales involved in the vertical gradients of constituents are larger than the scale of the turbulence. Some measurements made in the ocean show that the classical concept does not work at all in many cases (Moum 1990). Besides, on a global scale, mixing may occur through coherent motions rather than random motions. In this way, upwelling in equatorial region at equinox could be a significant factor in levitating cloud particles and supplying water vapor and deposition nuclei from below, as it could be the case for terrestrial noctilucent clouds (McIntyre 1989), called NLC in the following. There are analogies between high Martian clouds and NLC, formed at high altitude $(80-90 \mathrm{~km})$ near the very cold polar summer mesopause $(130-140 \mathrm{~K})$ : both form at low pressure and low temperature, and both appear as thin layers. In the case of the Earth, upwelling velocities of several centimeters per second, with strong adiabatic cooling, are expected above summer polar regions. Such motions are due to the pole to pole circulation induced by breaking gravity waves, with a drag effect on the mesospheric easterly summer and westerly winter jets inducing momentum transport. One of the most spectacular effects is a reversing of the pole to pole thermal gradient with a hot winter and a cold summer mesopause. Temperature inversions, due to the adiabatic compression of air moving downward, are observed and modeled at mid and high latitudes in the winter hemisphere (Hauchecorne and Maillard 1990). A pole to pole circulation can be also inferred from the retrograde zonal circulation observed in the Mars' middle atmosphere near northern winter solstice from Doppler-shifted CO lines in the microwaves range (Lellouch et al. 1991), suggesting a pattern rather similar to the terrestrial mesospheric circulation. A more specific theoretical work by Barnes (1990) shows that breaking gravity waves could have the same effect as on Earth at high latitudes. At Martian equinox, equator to pole circulation is expected, as shown for example by Moreau et al. (1991; called MEB hereafter). Adiabatic cooling and heating above respectively equatorial (rising branch of the Hadley cell) and polar (descending branch) regions may be seen in Fig. 4a of MEB. Fig. 4c of MEB is a clear illustration of the equator to pole circulation near the northern spring equinox. Assuming that upwelling (with an ascending velocity $U$ ) is the major recycling process, it is not possible to write explicitly a rigorous zero flux equation [like Eq. (11)] because of the nonsteady state, water vapor being continuously brought from below and transported toward the pole under vapor and/or ice form. Nevertheless, assuming a rough balance between the upward flux of water vapor $\left[U n_{\mathrm{g}}\right]$ and the downward flux of ice $\left[\left(V_{\mathrm{set}}-U\right) n_{\mathrm{s}}\right]$ inside the cloud and using the analytical from of $n_{\mathrm{g}}$ [Eq. (12)], one may derive to the first approximation, valid in the region above the cloud peak:

$$
n_{\mathrm{s}} \approx n_{\mathrm{g}_{0}} \frac{U}{V_{\mathrm{set}}-U} \exp \left(-\frac{\gamma}{\alpha}\left(z-z_{0}\right)\right)
$$

i.e., a kind of dependence very similar to what is obtained in the case of a low $K$ value [Eq. (17)], with a small scale height of $\alpha / \gamma(2-3 \mathrm{~km})$, therefore a very thin cloud. Because $U$, as $V_{\text {sel }}$, is expected to be inversely proportional to the atmospheric density (McIntyre 1989), Eq. (18) is approximately valid everywhere in the cloud. Comparing Eqs. (17) and (18), it may be inferred to the first approximation that the upwelling velocity required for efficient recycling is

$$
U_{\mathrm{eq}} \approx K_{\mathrm{eq}} \frac{\gamma}{\alpha} \approx 1-3 \mathrm{~cm} \mathrm{sec}^{-1} .
$$

An upward velocity of some centimeters per second, 
which is quite plausible, could be sufficient to carry up water vapor and condensation nuclei, counteract settling of ice particles, and therefore levitate clouds at high altitude. The formation of equatorial clouds would take place near the top of the rising branch of the Hadley cell, even in the absence of significant eddy mixing. An intermediate solution should be that eddy mixing and upwelling both contribute to replenish water vapor and dust from below. At lower altitudes, the upward velocity, which is inversely proportional to the atmospheric density, must be quite low and the $K$ value derived from the analysis of the dust is less questionable.

The temperature at $\sim 50 \mathrm{~km}$ altitude deduced from the present analysis of the clouds $(\sim 170 \mathrm{~K})$ is about $20 \mathrm{~K}$ above its generally assumed value $(\sim 150 \mathrm{~K})$, derived from models (Pollack et al. 1990, Fig. 3c; MEB, Fig. 4a) or observations, e.g., those of Viking landers (Seiff and Kirk 1977). In the rising branch of the Hadley cell, adiabatic cooling of ascending air parcels is expected to occur and clouds should be formed definitely lower than observed. The possible existence of two condensation levels, at respectively $\sim 15 \mathrm{~km}$ and $\sim 50 \mathrm{~km}$ altitude, related to a very specific thermal structure with an inversion layer between 35 and $50 \mathrm{~km}$ altitude, is discussed elsewhere (Chassefière and Blamont 1992). It must be noted that the model of MEB does not forecast any upward motion at $50 \mathrm{~km}$ altitude and $0^{\circ}-20^{\circ}$ north latitude near northern spring equinox due to a marked asymmetry of Hadley cells. Strong upwelling is therefore doubtful. Concerning clouds observed in Viking images, nothing certain can be concluded: the two lowest clouds, observed at nearly opposite latitudes $\left(60^{\circ} \mathrm{N}\right.$ and $\left.-45^{\circ} \mathrm{S}\right)$ after northern summer solstice when a north to south pole circulation is expected are rather similar despite the fact that vertical motions might occur in opposite directions. The role of convection in creating the clouds observed from Phobos and Viking is therefore far from established. We conclude that the $K_{\text {eq }}$ derived here (Fig. 18) represents approximately the eddy mixing coefficient, although upwelling could in principle contribute for a substantial part to the upward transport of water and dust in the high atmosphere.

\section{Consequences for Photochemistry}

Aerosols and eddy mixing could play a critical role in the stability of the Martian $\mathrm{CO}_{2}$. The presence of ice hazes to relatively high altitudes in the Martian atmosphere discussed in this paper offers an attractive possibility for recycling $\mathrm{CO}_{2}$. In general, aerosols of both dust and ice can play an important role in maintaining the stability of the atmosphere of Mars. The classic problem of the stability is as follows: in the absence of an effective recycling mechanism, all of the $\mathrm{CO}_{2}$ in the atmosphere of Mars should have been destroyed in less than 10,000 years, and the present day levels of $\mathrm{CO}$ and $\mathrm{O}_{2}$ (about $0.1 \%$ each) should have been attained in less than 3-6 years. Yet, neither is $\mathrm{CO}_{2}$ found to vary (except for regular seasonal cycles), nor do $\mathrm{CO}$ and $\mathrm{O}_{2}$ continue to build up. Mechanisms proposed in the pre-Viking era relied on catalytic oxidation of $\mathrm{CO}$ on $\mathrm{OH}$. One mechanism suffered from the requirement of unacceptably large values of the eddy mixing coefficient $\left(K>10^{8} \mathrm{~cm}^{2} \mathrm{sec}^{-1}\right)$ in the middle atmosphere (McElroy and Donahue 1972), while the other required an unrealistically large mixing ratio of water vapor, $\sim 1000 \mathrm{ppm}$ or $50 \mathrm{pr} . \mu \mathrm{m}$ (Parkinson and Hunten 1972). Large values of $K$ are expected during dust storms and in the high atmosphere, and the McElroy-Donahue scheme could play an important role on a local scale. The second mechanism, which may be expected to play an important role on a global scale, might work with reasonable amounts of water vapor (Shimazaki 1989). But, as suggested by this author himself, and by Chassefière (1991) in a detailed analysis of mechanisms regulating $\mathrm{CO}$, it is impossible at the present time to produce a suitable theoretical value of the $\mathrm{CO}$ abundance using a one-dimensional model. Due to the weakness of the processes regulating $\mathrm{CO}$, its equilibrium abundance is extremely unstable, although its long lifetime prevents large latitudinal or temporal variations. Therefore it is important to investigate the processes which might contribute to the recycling of $\mathrm{CO}_{2}$, in particular the possible role of heterogeneous chemistry.

A recent hypothesis proposed by Atreya and Blamont (1990) involves oxidation of $\mathrm{CO}$ on aerosols. It differs from earlier suggestions (e.g., Kong and McElroy 1977) in that the heterogeneous process occurs in the atmosphere rather than on the surface of Mars. Such catalysis in the atmosphere would alleviate the difficulty of having to transport species, such as $\mathrm{O}$, rapidly (e.g., by $K>10^{8} \mathrm{~cm}^{2}$ $\left.\sec ^{-1}\right)$ to the surface since the aerosols are present where the species are produced. It is also important to recognize that the surface area provided by aerosols is comparable to the local area of the surface of Mars. Aerosols above refer to airborne particles of both dust and ice. They can serve simply as convenient surfaces in the atmosphere for heterogeneous processes to proceed, or they could actually participate chemically in the heterogeneous catalysis. In the following these two possibilities are explored briefly.

Should the aerosols act simply as surfaces on which $\mathrm{CO}$ could oxidize, the rate of loss of $\mathrm{CO}$ on aerosols must be comparable to the only other loss process for this species, the most important of which is

$$
\mathrm{CO}+\mathrm{OH} \rightarrow \mathrm{CO}_{2}+\mathrm{H}
$$

Recycling of $\mathrm{CO}_{2}$ by (R1) suffers from the difficulties mentioned earlier. Using our measurements of the density 
$n\left(\sim 1 \mathrm{~cm}^{-3}\right.$ at $\left.\sim 20 \mathrm{~km}\right)$ and the effective radius $r(\sim 1-1.5$ $\mu \mathrm{m})$ of the dust, the collision rate of $\mathrm{CO}$ on dust aerosols may be derived by multiplying the $\mathrm{CO}$ number density by the collision frequency $n \pi r^{2} V_{\mathrm{T}}$, where $V_{\mathrm{T}}$ is the thermal velocity of the $\mathrm{CO}$ molecules. It is $\sim 3 \times 10^{8} \mathrm{~cm}^{-3} \mathrm{sec}^{-1}$, yielding a loss rate of $\mathrm{CO}$ on the dust of $\sim 3 \times 10^{4} \mathrm{~cm}^{-3}$ $\mathrm{sec}^{-1}$, which is of the same order as for R1 $\left(\sim 10^{5} \mathrm{~cm}^{-3}\right.$ $\mathrm{sec}^{-1}$ for $K \approx 10^{6} \mathrm{~cm}^{2} \mathrm{sec}^{-1}$ ). The accommodation coefficient of $\mathrm{CO}$ an anatase $\left(\mathrm{TiO}_{2}\right)$ is taken to be $\sim 10^{-4}$ from recent measurements at JPL (Leu et al. 1992). It is also assumed that $\mathrm{TiO}_{2}$ is present in the airborne dust in the same proportion as in the surface crust, i.e., $\sim 1 \%$. Although the dust is expected to contain oxides of iron also, the sticking coefficients of $\mathrm{CO}$ on hematite and magnetite are found to be orders of magnitude lower than on $\mathrm{TiO}_{2}$. Laboratory measurements further show that oxidation of $\mathrm{CO}$ does occur on $\mathrm{TiO}_{2}$ at long ultraviolet wavelengths (Thevenet et al. 1974):

$$
\mathrm{CO}+\mathrm{O}+\mathrm{TiO}_{2} \stackrel{210-390 \mathrm{~nm}}{\longrightarrow} \mathrm{CO}_{2}+\mathrm{TiO}_{2}
$$

The vertical distribution of aerosols in equatorial regions around the northern spring equinox, as sounded by our instrument, may be considered as the less favorable situation for heterogeneous chemistry. The atmosphere is clear above $25 \mathrm{~km}$ altitude, where no dust in appreciable amounts is present, and clouds are rather diffuse, with very small vertical optical depths. From Mariner 9 observations, it is known that during global storms the dust extends up to $\sim 40-50 \mathrm{~km}$ altitude with optical depths much larger than unity. The present study shows that under normal conditions, at the time of Phobos observations, the airborne dust can provide the surfaces responsible for heterogeneous processes to occur up to an altitude of approximately $25 \mathrm{~km}$. Higher in the atmosphere, the aerosols will be mostly in the form of ice particles. In fact, ice particles are expected to participate in the heterogeneous process down to $15 \mathrm{~km}$, as they are usually present in that height, and sometimes all the way to the surface. Nevertheless, they could be less efficient than dust particles, at least under normal conditions. The surface of dust per square centimeter of column is about $1 \mathrm{~cm}^{2}$, whereas ice particles offer only $1 \mathrm{~mm}^{2}$ per $\mathrm{cm}^{2}$ on average (see Table II). A rough calculation shows that a $\mathrm{CO}$ molecule collides with an ice particle of the high clouds observed from Phobos only a few times per year, implying that sticking is very rare and requires a time much larger than the photochemical lifetime of $\mathrm{CO}$. For this reason, ice particles might play a significant role only at some places or times of the seasonal cycle, whereas dust could participate in heterogeneous chemistry all the time.

Since $\mathrm{CO}$ has almost no dipole moment and $\mathrm{O}$ is nonpolar, neither is soluble in water ice, thus the possibility of any $\mathrm{CO}$ oxidation on pure particles is very remote. The ice particles would be most effective in the heterogeneous chemical catalysis if they were doped with certain Martian constituents, such as $\mathrm{NO}_{x}$ or chlorine. The surface composition of Mars indicates chlorine to be present at a level of $\sim 1 \%$ and it may be present in the atmosphere as well, e.g., it may be carried along with the dust. $\mathrm{NO}_{x}$ have not been detected on Mars; however, considering the photochemical processes occurring on Mars, it is inevitable that they would be present at least at the $10^{-2}$ to 1 ppm level. It has also been suggested that the odd nitrogen species, such as $\mathrm{HNO}_{3}, \mathrm{HNO}_{2}, \mathrm{HO}_{2} \mathrm{NO}_{2}, \mathrm{NO}_{2}$, and $\mathrm{N}_{2} \mathrm{O}_{5}$ may be scavenged by ice aerosols in the atmosphere of Mars (Y. L. Yung, 1990, personal communication). The measurement capabilities of the instruments on Mars missions so far could not permit detection of odd chlorine or odd nitrogen constituents less than $\sim 10^{-6}$ mixing ratio. For heterogeneous chemical oxidation of $\mathrm{CO}$ on ice aerosols, one requires only $10^{-7}$ to $10^{-8}$ for the mixing ratios of chlorine or odd nitrogen.

The validity of the above-mentioned hypothesis of oxidation of $\mathrm{CO}$ by heterogeneous processes on aerosols can be tested fully only after additional laboratory data on appropriate sticking coefficients, along with their temperature dependence, and observational results on temporal and spatial distributions of $\mathrm{H}_{2} \mathrm{O}, \mathrm{CO}, \mathrm{O}_{3}$, and $\mathrm{O}_{2}$, as well as chlorine and odd nitrogen constituents, become available. There is much hope of resolving the issue of the stability of the Martian atmosphere as well as of the possible "COhole" (Rosenquist et al. 1990) following the observations of Mars 1994/1996 (SPICAM and PFS instruments) and Mars Observer (PMIRR and TES instruments).

\section{APPENDIX A}

In Fig. Al are plotted the photon fluxes detected at 1.9 and $3.7 \mu \mathrm{m}$, the transmission in the $\mathrm{CO}_{2}$ band and the pointing flux in the upper eye of the $H$ axis (see Fig. 2 in PSP) as a function of the altitude of the IR line of sight, for the first type 2 occultation (S221). Signals at 1.9 and 3.7 $\mu \mathrm{m}$ are obtained by averaging the signal over the whole spectral range of the relevant channels. Absorption by water vapor at $5360 \mathrm{~cm}^{-1}$ does not exceed 2 or $3 \%$ above $15 \mathrm{~km}$ (Krasnopolsky ef al. 1991) and the corresponding pixel is not removed since it does not affect flux at 1.9 $\mu \mathrm{m}$ by more than one-thousandth. Because of thermal problems, it is not possible to use spectra obtained outside of the atmosphere, about $10 \mathrm{~min}$ before occultation, as a reference. It was decided to use the first spectrum of the occultation sequence. The $\mathrm{CO}_{2}$ transmission at the highest sounded altitude is 1 by definition. As explained in PSP, we are able to reconstruct the series of altitudes apart from an unknown vertical translation. The absolute altitude depends mainly on the vertical optical depth $\tau$ of the dust. The altitude reported in Fig. A1 was obtained initially by assuming $\tau=0.3$ in the simulation algorithm but it is now corrected according to the analysis of the $\mathrm{CO}_{2}$ profile, as explained in the next paragraph. There is a rapid decrease of the IR fluxes below $\sim 25 \mathrm{~km}$ which may be attributed without ambiguity to the dust component of the atmosphere. The level at which the horizontal optical thickness at $1.9 \mu \mathrm{m}$ is 0.1 is indicated by a horizontal dotted line. The stability of the 

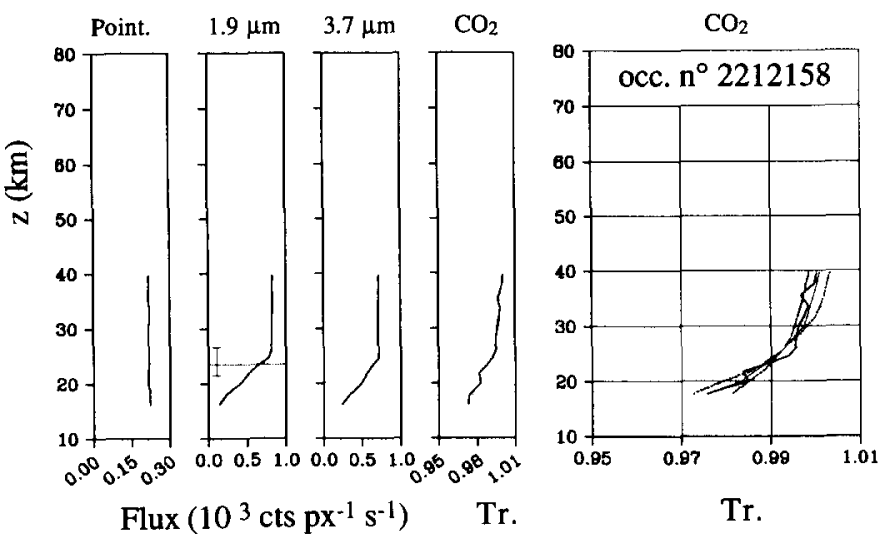

FIG. A1. From the left to the right are plotted as a function of the altitude of the IR line of sight (S221): (1) the pointing flux in the highest eye of the $\mathrm{H}$ axis in units of $10^{3}$ counts per second; (2) the integrated flux at $1.9 \mu \mathrm{m}$ in units of $10^{3}$ counts per second and per pixel; (3) the integrated flux at $3.7 \mu \mathrm{m}$ in the same units; (4) the transmission in the $\mathrm{CO}_{2}$ band at $5313 \mathrm{~cm}^{-1} ;(5)$ the mean and extreme theoretical $\mathrm{CO}_{2}$ profiles (dotted lines) compared to the observed one (solid line). The 0.1 horizontal optical depth level at $1.9 \mu \mathrm{m}$ is indicated by an horizontal dotted line, as well as the error bar on the altitude.

pointing flux proves that the pointing system works in the nominal way. As seen in Fig. A2 (S245), the pointing is less good for later occultations, being progressively lost below the 0.1 optical depth level. During S245, a cloud was observed around $50 \mathrm{~km}$ altitude in the UV and NIR channels (see the Section The Data). This is the only cloud observed in type 2.

Using the same calculations of the $\mathrm{CO}_{2}$ band absorption as Krasnopolsky et al. (1991), the unknown vertical translation $z_{0}$ to be applied to the series of altitudes $z(t)$ is determined by minimizing the deviation between the observed $\mathrm{CO}_{2}$ transmission profile and the theoretical one. The calculation of the $\mathrm{CO}_{2}$ band that we use in the present work is based on a simplified parametrization of the Voigt profiles. As noted by Krasnopolsky et al. (1991), it is in good agreement with other existing calculations. A more detailed spectral analysis is being performed at the present time by one of us (O. I. Korablev) in order to improve the estimate of some other minor constituents but it cannot affect significantly the $\mathrm{CO}_{2}$
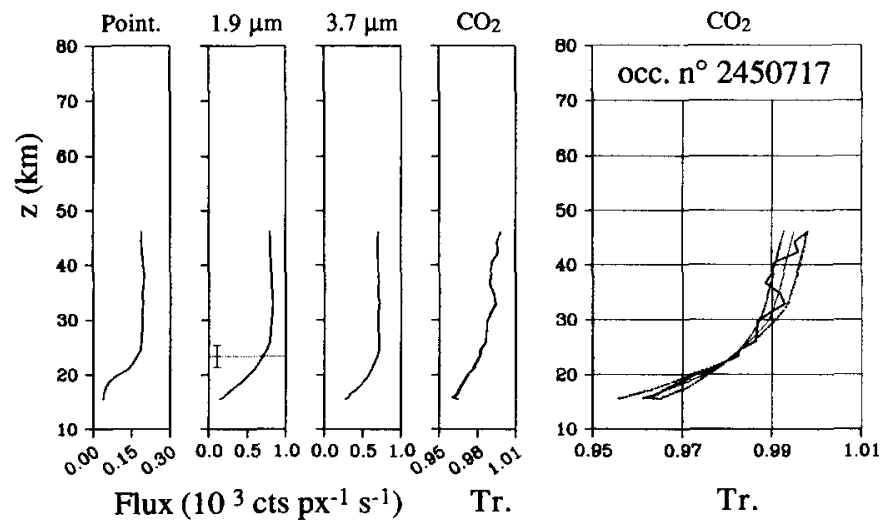

Tr. absorption and therefore the retrieved altitude. In order to determine the most likely value of $z_{0}$, with error bars, the theoretical and the observed profiles are scaled together and the root of the mean square deviation $d\left(z_{0}\right)$ is calculated for one hundred values of $z_{0}$ (between -20 $\mathrm{km}$ and $+80 \mathrm{~km}$ ). The scaling is made necessary by the fact that the $\mathrm{CO}_{2}$ transmission is given relative to the one at the highest sounded altitude, which is not 1 except in some cases of type 1 occultations beginning at a very high altitude. The most likely value of $z_{0}$ is obtained by minimizing $d\left(z_{0}\right)$. Assuming Gaussian residuals, an error bar may be defined at the $2 \sigma$ level. Because this definition of the uncertainty is in the present case rather artificial, we present in Figs. Al and A2 (right side) the most likely and the two extreme theoretical $\mathrm{CO}_{2}$ transmission profiles fitting the observed one and let the reader judge. The error bar on the altitude is indicated on the 1.9- $\mu \mathrm{m}$ flux curve. Corresponding extreme $\mathrm{CO}_{2}$ transmission profiles are put back to the altitude of the mean profile and plotted for comparison.

\section{APPENDIX B}

A first examination of type 1 occultations shows an incomprehensible behavior of the IR signal. The minimum altitude reached by the IR line of sight is about $10 \mathrm{~km}$ above the one reached for the type 2, suggesting that the very beginning of the occultation phase might have been observed. Instead, the signal has a quite erratic variation when the loss of pointing occurs, remaining at a nearly constant level in some cases, increasing or decreasing in other cases without any clear tendancy. In one case (S230), there is a sharp increase of the IR fluxes and the $\mathrm{CO}_{2}$ transmission when the loss of pointing occurs.

In Fig. B1 are plotted for S230 the photon fluxes at 1.9 and $3.7 \mu \mathrm{m}$, the transmission in the $\mathrm{CO}_{2}$ band, and the pointing flux in the eye opposite to $L$ on the $V$ axis (see Fig. 2 in PSP), i.e., the highest eye, as a function of altitude. The altitude reported in Fig. B1 is obtained without any correction, assuming $\tau=0.3$ in the simulation algorithm. It is defined apart from an unknown constant and may be therefore shifted upward or downward depending on the exact location of the top of the dark layer. The decrease of the pointing flux below $\sim 27 \mathrm{~km}$ indicates that the descent velocity of the line of sight progressively decreases, the highest eye moving outward from the solar disk with a subsequent decrease of the flux. This drift is produced by a darkening of the atmosphere in the field of view of the lowest eye $L$. In principle, a decrease of IR fluxes is expected to occur at the time of the drift since the IR line of sight is at nearly the same altitude as $L$, instead of the observed increase. The only
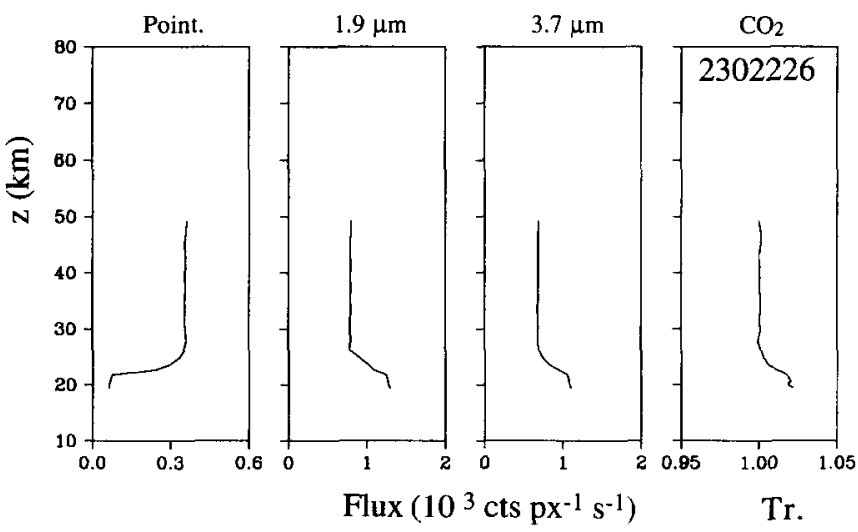

FIG. B1. Same as the left parts of Figs. A1 and A2 for S230. There is a clear anticorrelation between the pointing flux and the three other quantities.

FIG. A2. Same as Fig. A1 for S245. 
way to explain this unexpected behavior is to assume that the IR field is shifted with respect to its nominal position and intersects the edge of the Sun. When the descent velocity begins to slow down, the field progressively enters the Sun with a subsequent increase of the flux masking the expected decrease due to atmospheric darkening. The solar limb darkening effect cannot explain this phenomenon because it is very weak in infrared. It is more difficult to explain the increase of the $\mathrm{CO}_{2}$ transmission since this quantity is obtained as a ratio of the average transmission on three consecutive pixels to the average on adjacent pixels. The observed increase implies that there is a deformation of the spectrum at $1.9 \mu \mathrm{m}$ when the loss of pointing occurs. As previously mentioned, this kind of phenomenon is also observed in the UV due to the combination of limb darkening and a parasitic reflection inside the instrument. One may suspect the same kind of effect in the IR subsystem, although no satisfactory explanation has been found at the present time.

Let us first consider fluxes at 1.9 and $3.7 \mu \mathrm{m}$. Because of the anticorrelation between the pointing flux and the IR fluxes, as shown in Fig. B1, the most natural way to take into account this effect is to monitor instrumental variations of the signal by the pointing flux in the highest eye, according to S230: for each occultation, the theoretical evolution of the IR fluxes in the absence of atmospheric effects may be calculated by applying to the pointing fluxes the relationships derived from those observed for $S 230$ (Fig. B1). Comparing the observed and the predicted evolutions, one may in principle isolate atmospheric extinction. A priori, this approach is not correct since there is likely some atmosphere extinction attenuating the final increase of the signal during S230. This is proven by the fact that for all type 1 occultations, the signal remains nearly constant, or in some cases weakly decreases (or increases). For several reasons, we think that the anomalous behavior of S230 is due to the presence of a thick cloud at high altitude which interfered with the pointing system and was not penetrated by the line of sight. Pointing simulations show that the most prominent cloud observed by our instrument (S236: see Fig. 3 and 4 in PSP) nearly blocked the line of sight and the presence of such a cloud at the time of $S 230$, just slightly thicker than the cloud observed during S236, is plausible. The strongest argument in favor of this hypothesis is the flatness of the $\mathrm{CO}_{2}$ transmission profile prior to the loss of pointing, contrary to the regular decrease observed in other cases, which seems to show that the part of the at mosphere sounded by the IR line of sight is still at high altitude, $\sim 40-50 \mathrm{~km}$ above values indicated on the ordinate axis of Fig. B1. As shown in the next section, the scale height of the extinction coefficient in the upper part of the cloud is extremely small ( 2 or $3 \mathrm{~km}$ ), about one-half of the dust extinction scale height around $20 \mathrm{~km}$ altitude, which is not surprising. Because of the sharpness of the upper edge of the cloud, atmospheric extinction occurs later than in other cases, revealing the instrumental effect.

As suspected, the direct use of relationships derived from Fig. B1 to correct IR fluxes does not give satisfactory results. Because complete infrared occultations are performed in nine cases (type 2), from which vertical profiles of dust extinction at 1.9 and $3.7 \mu \mathrm{m}$ are obtained without ambiguity, one may compare extinction profiles obtained for type 1 applying the pointing correction to those obtained for type 2. For type 1 , the general picture is completely modified when taking into account pointing effects, in the way previously explained, and becomes more consistent with type 2 observations. Instead of a constant or weakly varying signal when the loss of pointing occurs, a sharp inflexion appears with a rapid decrease of the signal below. The top of the dust opaque layer is found at $\sim 25 \mathrm{~km}$ altitude, consistent with type 2 results. Nevertheless, the slope of the decrease just below the lop of the dust opaque layer is too low, especially at $1.9 \mu \mathrm{m}$. Therefore the increase of the signal observed at $1.9 \mu \mathrm{m}$ when the loss of pointing occurs for S230 (Fig. B1) is very likely underestimated. This is not surprising since, as previously mentioned, some atmospheric extinction must temper the instrumental increase. In view of this fact, relationships derived from Fig. B1 have been modified in such a way to obtain a better general agreement between type 2 and type 1 dust profiles. Other criteria have
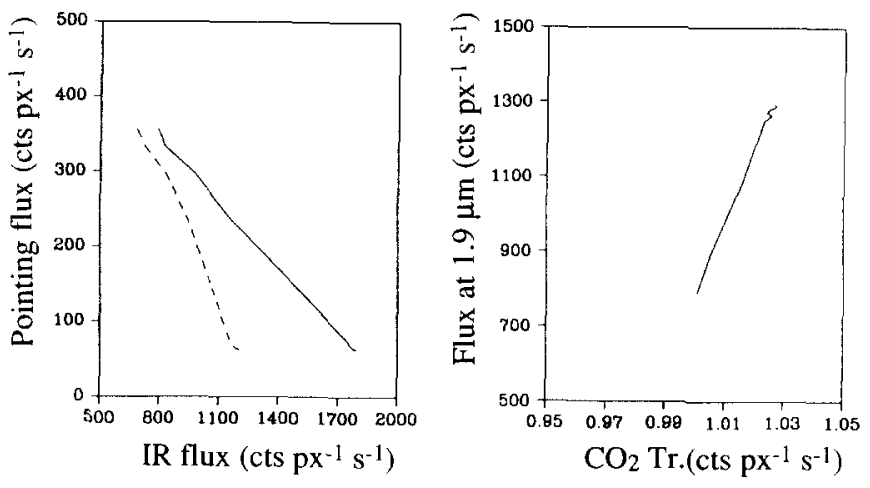

FIG. B2. On the left are plotted the relationships between the pointing flux and the photon fluxes at $1.9 \mu \mathrm{m}$ and $3.7 \mu \mathrm{m}$ (resp., solid and dashed line) in the absence of atmospheric effects. On the right is plotted the relationship between the flux at $1.9 \mu \mathrm{m}$ and the transmission in the $\mathrm{CO}_{2}$ band.

also been taken into account, like obtaining a better flatness for profiles in the clear atmosphere or smoothing the sharp changes of slope observed in some cases when the pointing is severely disturbed. Relationships finally adopted are indicated in Fig. $\mathrm{B} 2$ (left side). For $\mathrm{CO}_{2}$, an extensive study of all type 1 occultations shows without ambiguity that the increase of the transmission in the $\mathrm{CO}_{2}$ band at the end of $\mathrm{S} 230$ (Fig. B1) must be correlated to the flux at $1.9 \mu \mathrm{m}$, not to the pointing flux. The deformation of the spectrum is not linked to a particular geometry when the loss of pointing occurs but simply to the level of the signal, which may be altered by both atmospheric and/or instrumental effects. For $\mathrm{CO}_{2}$, we have adopted, without correction, the relationship derived from Fig. B1 (Fig. B2: left part).

One tricky point is the nonproportionality between fluxes at $1.9 \mu \mathrm{m}$ and $3.7 \mu \mathrm{m}$ deduced from the relationships shown in Fig. B2, which contradicts to some extent the interpretation previously proposed (that the IR field of view intersects the edge of the Sun under the nominal pointing conditions). Our choice of corrective functions may therefore seem unrealistic. In fact, a definite nonlinearity is already observed prior to the correction procedure in a lot of cases. From an examination of the whole set of type 1 occultations, it may be seen that the ratio $R$ of the $1.9-\mu \mathrm{m}$ flux to the $3.7-\mu \mathrm{m}$ flux when the loss of pointing occurs either remains nearly constant or increases with a rate varying from one occultation to another. In the case of S230 (Fig. B1), the increase is quite small $(\sim+8 \%)$ but it may reach $20 \%$ in some cases (e.g., for S243). This increase may not be attributed to an atmospheric effect because expected micrometer-sized particles are more transparent at $3.7 \mu \mathrm{m}$ than at 1.9 $\mu \mathrm{m}$. Atmospheric extinction might therefore produce the inverse feature (a decrease of $R$ ), which proves that the increase of $R$ deduced from untreated data when the loss of pointing occurs is still underestimated. The variation of $R$ by $\sim 40 \%$ deduced from Fig. B2 is therefore not qualitatively different than instrumental variations observed for data (up to $20 \%$ ), and may potentially explain why atmospheric extinction is never seen directly in the IR channels. The nonproportionality between the two IR fluxes is a mystery. The same kind of effect is observed in the $\mathrm{UV}$ when the pointing is lost, mainly because the line of sight moves on to the external parts of the solar disk, and is now perfectly understood. Due to the decentered position of the IR line of sight, probably straddling the edge of the Sun, an unusual behavior cannot be excluded. For example, any little misalignment of detectors could involve unexpected variations of the signal and of its spectrum if the Sun fills only one part of the field

Figure B3, similar to Figs. A1 and A2, shows the vertical variation of 

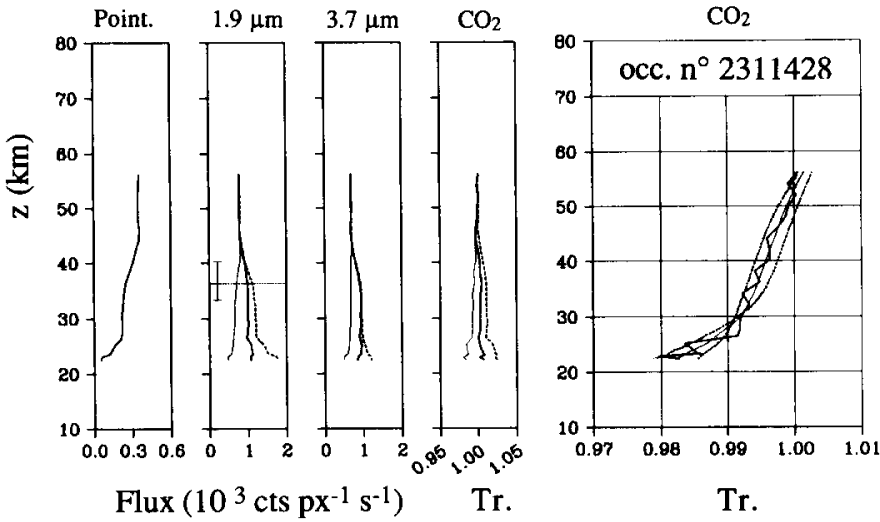

FIG. B3. Same as Figs. A1 and A2 for the type 1 occultation S231. The observed IR fluxes and $\mathrm{CO}_{2}$ transmission (thick solid lines) are corrected from the instrumental profile (dashed lines) obtained applying functions defined in Fig. B2 to the pointing flux. The thin solid lines indicate the corrected profiles.

the IR fluxes for the type 1 occultation S231. In this case, the signal is corrected using functions given in Fig. B2. S231 is a classical type 1, except that a cloud is detected in the UV and the NIR around $50 \mathrm{~km}$ altitude. This cloud does not appear at infrared wavelengths because it is weak and particles are small. The thick solid line on the IR flux and $\mathrm{CO}_{2}$ transmission plots is the untreated signal. The anticorrelation shown in Fig. $\mathrm{Bl}$ between the pointing flux and the other quantities is quite apparent. The dashed line is the instrumental signal reconstructed from the pointing flux using relationships plotted in Fig. B2, i.e., the signal which would be observed in the absence of atmospheric effects. Thin solid lines are obtained as the ratio of the observed signal to the instrumental one and are thought to be representative of purely atmospheric effects. The correction procedure removes the instrumental increase observed when the pointing flux decreases and provides a good continuity of profiles from 50 to $30 \mathrm{~km}$. The unusual length of the loss of pointing phase, extending from 45 to $25 \mathrm{~km}$, may be attributed without ambiguity to a tenuous absorbing (or scattering) layer in the $15 \mathrm{~km}$ above 25 $\mathrm{km}$ (top of the dust opaque layer under the usual conditions). This is confirmed by the profile at $1.9 \mu \mathrm{m}$, which shows a definite extinction below $40 \mathrm{~km}$ altitude, slowly increasing down to $25 \mathrm{~km}$. Extinction is much less apparent at $3.7 \mu \mathrm{m}$. The altitude is rather well defined from $\mathrm{CO}_{2}$ absorption, with a larger error bar than for type 2 because the altitude is higher and the $\mathrm{CO}_{2}$ absorption is smaller ( $\sim 2 \%$ for $\mathrm{S} 231$, against $\sim 3 \%$ for $\mathrm{S} 221$ and $\sim 4 \%$ for $\mathrm{S} 245$ at the bottom).

For the two darkest clouds observed by our instrument (S236 and S251; see PSP), the pointing is so disturbed by the presence of a cloud that it is more difficult to estimate the altitude. A lower limit on $z_{0}$ may be applied with confidence, but we cannot give an upper limit because the altitude is high and therefore the $\mathrm{CO}_{2}$ absorption is weak. The instrumental variations of the signal (especially for S236) are so sharp that a small error on corrective functions implies a large error on the $\mathrm{CO}_{2}$ absorption. There is not a perfect reproducibility of the relationship between the pointing and the IR fluxes from one occultation to the other, and all our attempts to change corrective functions in order to improve the treatment of S236 led to a general degradation of profiles. Figure B4 shows corrected profiles for S236. In both S236 and S251 cases, the clouds seen in the UV and the NIR are also observed in the IR, contrary to the cases of S245 (Fig. A2) and S231 (Fig. B3). The corrected $\mathrm{CO}_{2}$ transmission shows a large dispersion but the peak extinction level may be located with confidence above $53 \mathrm{~km}$ (S236) and $45 \mathrm{~km}$ (S251). No reliable upper limit can be proposed.
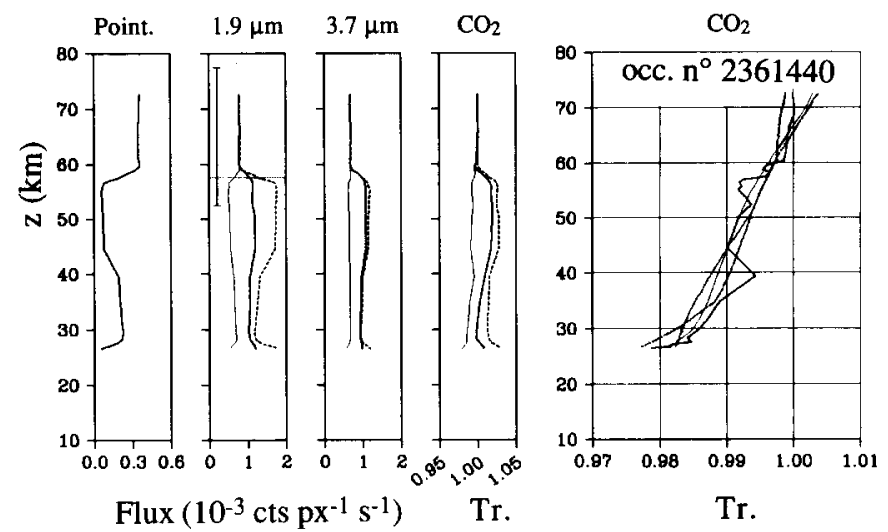

FIG. B4. Same as Fig. B3 for S236. Point is strongly disturbed by the presence of a thick cloud at $\sim 50 \mathrm{~km}$ altitude.

\section{APPENDIX C}

The extinction coefficient is assumed to be constant inside each observed layer $i$, defined as the altitude range sounded by the line of sight during the acquisition of one spectrum (Fig. C1). Let us denote by $\tau_{i}$ the tangentially integrated optical thickness of layer $i, p_{i}$, the impact parameter of the line of sight (distance from the lowest point of the line of sight to the center of Mars), $x_{i}$ and $l_{i j}$ the geometric lengths over which a line of sight of impact parameter $p_{i}$ intersects, respectively, layer $i$ and layer $j$, and $r_{i}$ the distance from the upper edge of layer $i$ to the center of Mars, the local extinction coefficient $\sigma_{i}$ may be derived step-by-step using the following scheme:

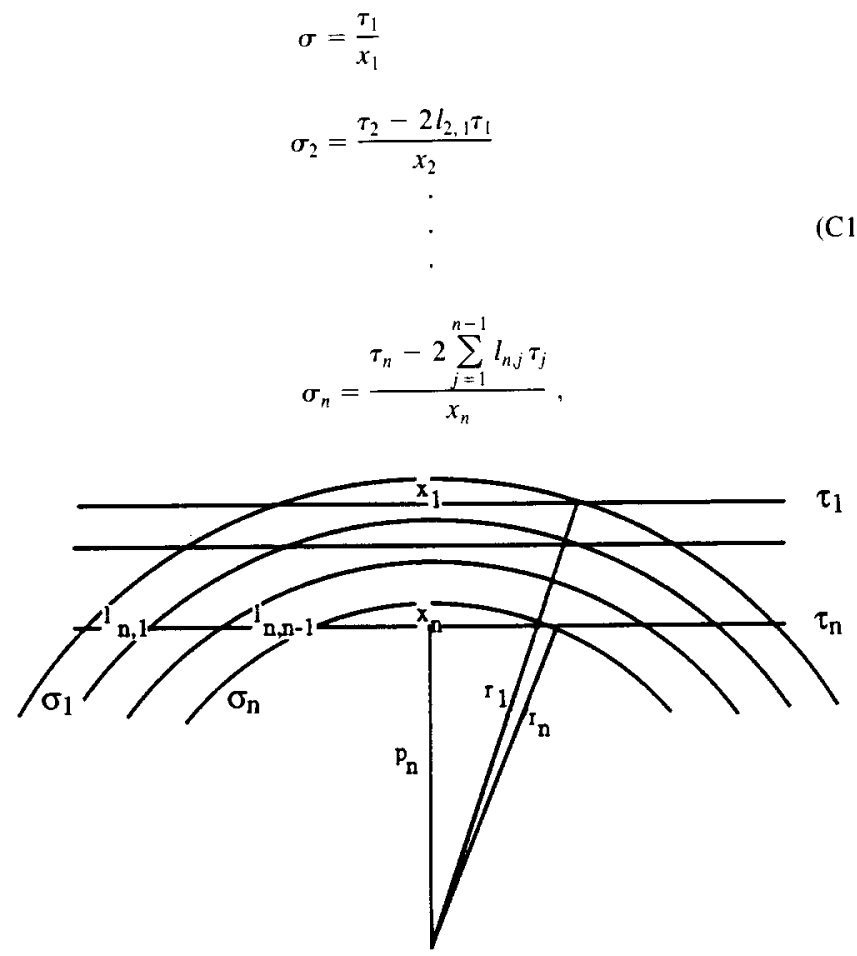

FIG. C1. Schematic view describing the principle of the "onion peeling" technique. 
where $n$ is the total number of layers and $x_{i}$, and $l_{i, j}$ are given by

$$
\begin{aligned}
& x_{i}=2 \sqrt{r_{i-1}^{2}-p_{i}^{2}} \\
& l_{i i}=\sqrt{r_{j}^{2}-p_{i}^{2}}-\sqrt{r_{j}^{2}-p_{i}^{2}} .
\end{aligned}
$$

\section{ACKNOWLEDGMENTS}

We are grateful to M. Nunes-Pinharanda for his detailed analysis of the spectra obtained in the NIR channels of the instrument, including fine corrections related to the integration time and the low signal to noise ratio. The present work would not have been possible without this critical phase of the data analysis which constitutes the central part of his thesis (Pinharanda 1990). S. K. Atreya acknowledges support received from NASA Grants NAGW-2561 and NAGW-1442. Part of this work was performed by the Jet Propulsion Laboratory, under contract with the National Aeronautics and Space Administration. We thank CNES for his continuous support of the Phobos project.

\section{REFERENCES}

Anderson, E. AND C. Leovy 1978. Mariner 9 television limb observations of dust and ice hazes on Mars. J. Almos. Sci. 35, 723-734.

Atreya, S. K., And J. E. Blamont 1990. Stability of the Martian atmosphere: Possible role of heterogeneous chemistry. Geophys. Res. Lett. 17(3), 287-290.

Barnes, J. R. 1990. Possible effects of breaking gravity waves on the circulation of the middle atmosphere of Mars. J. Geophys. Re's. B 95(2), 1401-1421.

Blamont, J. E., E. Chassefière, J. P. Goutall. B. Mège, M. Nunes-Pinharanda, G. Souchon, V. A. Krasnopolsky, A. A. KRYSKO, AND V. I. MOROz 1989. Vertical profiles of dust and ozone in the Martian atmosphere deduced from solar occultation measurements. Nature 341, 600-603.

Blamont, J. E., E. Chassefière, J. P. Goutall, B. Mège, M. Nunes-Pinharanda, G. Souchon, V. A. Krasnopolsky, A. A. Krysko, AND V. I. Moroz 1991. Vertical profiles of dust and ozone in the Martian atmosphere deduced from solar occultation measurements. Planet. Space Sci. 39, 175-187.

Chassefière, E. 1991. Photochemical regulation of CO on Mars. Geophys. Res. Lett. 18, 1055-1058.

Chassefière, E., J. Blamont, P. Drossart, J. Rosenqvist, M. Combes, S. Erard, Y. Langevin, and J. P. Bibring 1991. Vertical distribution and granulometry of Martian dust particles from the Phobos/ISM and Auguste experiments In 23rd Annual DPS Meeting.

Chassefière, E., AND J. E. Blamont 1992. Vertical transport of water ice at low latitudes in the Martian atmosphere. Geophys. Res. Lett., in press.

Clark, B. C., A. K. Baird, R. J. Weldon, D. M. Tsusaki, L. SchnaBEL, AND M. P. CANDELARIA 1982. Chemical composition of Martian fines. J. Geophys. Res. B 87(12), 10,059-10,067.

Colburn, D. S., J. B. Pollack, and R. M. Haberle 1989. Diurnal variations in optical depth at Mars. Icarus 79, 159-189.

HaNSEN, J. E., AND L. M. Travis 1974. Light scattering in planetary atmospheres. Space Sci. Rev. 16, 527-610.

Hauchecorne, A., And A. Maillard 1990. A 2-D dynamical model of mesospheric temperature inversions in winter. Geophys. Res. Lett. 17(12). 2197-3000.
JAKosky, B. M., AND C. B. FARmer 1982. Seasonal and global behavior of water vapor in the Mars atmosphere: Complete global results of the Viking atmosphere water detector experiment. J. Geophys. Res. 87, 2999-3019.

Jaquin, F., P. Gierasch, and R. Kahn 1986. The vertical structure of limb hazes in the Martian atmosphere. Icarus 68, 442-461.

KaHN, R. 1990. Ice haze, snow, and the Mars water cycle. J. Geophys. Res. B 95(9), 14,677-14,693.

KAHN, R. 1991. Ice hazes in the Martian atmosphere. In 20th General Assembly of the IUGG, IAMAP: The Climate of Mars, Vienna. M14.

KONG, T. Y., AND M. B. MCELRoY 1977. Photochemistry of the Martian atmosphere. Icarus 32, 168-189.

Krasnopolsky, V. 1986. Photochemistry of the Atmospheres of Mars and Venus. Springer-Verlag, New York/Berlin.

Krasnopolsky, V. A., V. I. Moroz, A. A. Krysko, O. I. Korabley. V. S. Zhegulev, A. V. Grigoriev, A. Yu. Tkachuk, V. A. Parshev, J. E. Blamont, AND J. P. Goutall 1989. Solar occultation spectroscopic measurements of the Martian atmosphere at 1.9 and 3.7 $\mu \mathrm{m}$. Nature 341, 603-604.

Krasnopolsky. V. A., O. 1. Korablev, V. I. Moroz, A. A. Krysko, J. E. Blamont, ANd E. Chassefière 1991. Infrared solar occultation sounding of the Martian atmosphere by the phobos spacecraft. Icarus 94, 32-44.

Lellouch, E., J, J. Goldstein, S. W. Bougher, G. Paubert, and $\mathrm{J}$. ROSENQVIST 1991. Absolute wind measurements in the middle atmosphere of Mars. Ap. J. 383, 401-406.

Leu, M. T., J. E. Blamont, A. Anbar, L. F. Keyser, and S. P. SANDER 1992. Sticking coefficients of CO on aerosols: Implications for Martian atmosphere. J. Geophys. Res. C97(3), 2621-2627.

Mason, B. J. 1971. The Physics of Clouds, p. 108. Clarendon Press, Oxford.

McElroy, M. B., AND T. M. Donahue 1972. Stability of the Martian atmosphere. Science 177, 986-988.

MCINTYRF, M. E. 1989. On dynamics and transport near the polar mesopause in summer. J. Geophys. Res. D. 94(12), 14,617-14,628.

Michelangeli, D. V., O. B. Toon, R. Haberle, and J, B. Pollack 1992. Numerical simulations of the formation and evolution of waterice clouds in the Martian atmosphere. Submitted for publication.

Moreau, D., L. W. Esposito, and G. Brasseur 1991. The chemical composition of the dust-free Martian atmosphere: Preliminary results of a two-dimensional model. J. Geophys. Res. B. 96(5), 79337945.

Moum, J. N. 1990. The quest for $K_{\mathrm{p}}{ }^{-}$. Preliminary results from direct measurements of turbulent fluxes in the ocean. J. Phys. Ocean. 20, $1980-1984$.

Nunes-Pinharanda, M. 1990. Utilisation de la méthode des occultations solaires pour l'étude de l'atmosphère de Mars par l'instrument Auguste. Thèse d'Université.

PANG, K., AND J. M. AJEllo. 1977. Complex refractive index of Martian dust: Wavelength dependence and composition. Icartus 30, 63-74.

Parkinson, T. D.. AND D. M. HUNTEN 1972. Spectroscopy and aeronomy of $\mathrm{O}_{2}$ on Mars. J. Atmos. Sci. 29, 1380-1390.

Pollack, J. B., O. B. ToOn, and B. N. Khare 1973. Optical properties of some terrestrial rocks and glasses. Icarus 19, 372-389.

Pollack, J. B., D. S. Colburn, F. M. Flasar, R. Kahn, C. E. Carlston, and D. Pideck. 1979. Properties and effects of dust suspended in the Martian atmosphere. J. Geophys. Res. B. 84(6), 2929-2945.

Pollack, J. B., R. M. Haberle, J. Schaeffer, and H. Lee 1990. $J$. Geophys. Res. 95, 1447-1473. 
Rosenquist, J., J. P. Bibring, M. Combes, P. Drossart, T. ENcrenaz, S. Erard, O. Forni, B. Gondet, Y. Langevin, E. LelLOUCH, P. MASSON, AND A. SOUFLLOT 1990. The vertical distribution of CO on Mars from Phobos-ISM experiment. Astron. Astrophys. 231, L29-L32.

Rosenqvist, J., P. Drossart, M. Combes, T. Encrenaz, E. LelLoUCh, J. P. Bibring, S. ERARd, Y. LANGEVIN, ANd E. ChaSSEFIĖRE 1992. Minor constituents in the Martian atmosphere from the ISM/ Phobos experiment. Submitted for publication.

SEIFF, A., AND D. B. KIRK 1977. Structure of the atmosphere of Mars in summer at midlatitudes. J. Geophys. Res. 82(28), 43644378.

ShImAzaki, T. 1989. Photochemical stability of $\mathrm{CO}_{2}$ in the Martian atmosphere: Reevaluation of the eddy diffusion coefficient and the role of water vapor. J. Geomagn. Geoelectr. 41, 273-301.

Thevenet, A., F. Juillet, And S. J. Teichner 1974. Photointeraction on the surface of titanium dioxide between oxygen and carbon monoxide. Japan J. Appl. Phys. 2, 529-532.

WEST, R. A. 1992. Optical properties of aggregate particles whose outer diameter is comparable to the wavelength. Appl. Opt., 30(36), 5316-5324. 